\title{
Nonlinear Oscillations for Cyclic Movements in Human and Robotic Arms
}

\author{
Dominic Lakatos, Florian Petit, and Alin Albu-Schäffer
}

\begin{abstract}
The elastic energy storages in biologically inspired Variable Impedance Actuators (VIA) offer the capability to execute cyclic and/or explosive multi degree of freedom (DoF) motions efficiently. This paper studies the generation of cyclic motions for strongly nonlinear, underactuated multi DoF serial robotic arms. By experimental observations of human motor control, a simple and robust control law is deduced. This controller achieves intrinsic oscillatory motions by switching the motor position triggered by a joint torque threshold. Using the derived controller, the oscillatory behavior of human and robotic arms is analyzed in simulations and experiments. It is found that the existence of easily excitable oscillation modes strongly depends on the damping properties of the plant. If the intrinsic damping properties are such that oscillations excited in the undesired modes decay faster than in the desired mode, then multi-DoF oscillations are easily excitable. Simulations and experiments reveal that serially structured, elastic multi-body systems such as VIA or human arms with approximately equal joint damping, fulfill these requirements.
\end{abstract}

Index Terms-Nonlinear Oscillations, Variable Impedance Actuators, Underactuated Robots, Biologically-Inspired Robots, Motion Control.

\section{INTRODUCTION}

$\mathbf{C}$ YCLIC movements such as running or drumming or explosive motions such as throwing, hitting or jumping can be easily executed by humans. To approach athletic performance and efficiency, robot design evolved recently from classical, rigid actuation towards actuators with tunable intrinsic stiffness and/or damping, so called Variable Impedance Actuators (VIA). These elastically actuated robots are strongly inspired by the biological musculo-skeletal system [1]. They are motivated by biomechanics research which reveals the importance of the elasticity for robustness and energetic efficiency as well as for the maximization of peak force and velocity [2]. The goal is to achieve highly dynamic motions by exploiting intrinsic mechanical resonance effects of the systems. For very clearly defined tasks such as running, the hypothesis is that intrinsic oscillation modes of the mechanical system exist and correspond to meaningful gaits, which only need to be excited. Similar to the ideas of [3], where passive leg compliances are varied to optimize the gait speed, the stiffness variability of the VIA joints and the initial joint posture might be further used to shape these oscillation modes.

The generation of motor trajectories and the tuning of joint stiffness during highly dynamic motions are often addressed as an optimal control problem. While for single joints an analytical solution is feasible [4], [5], for the multi-joint

The authors are with the Institute of Robotics and Mechatronics, German Aerospace Center (DLR), D-82234 Oberpfaffenhofen, Germany \{dominic.lakatos, florian.petit, alin.albu-schaeffer\}@dlr.de. case numerical, multi-variable constrained optimizations need to be performed [6], [7]. The approach is currently limited to systems with few degrees of freedom (DoF). With an increasing number of degrees of freedom (for example in the case of a humanoid body ( $>30 \mathrm{DoF}$ ) or even one arm $(7 \mathrm{DoF})$ ) the computational complexity and the number of local minima explodes. A further related approach to control periodic running or swimming movements of systems with a large number of degrees of freedom is presented by Ijspeert et al. [8], [9] and [10]. As observed in neuro-control units of amphibians, periodic trajectories are generated by nonlinear (phase) coupled oscillators. The periodic motion replicating this behavior in a robotic system is generated in an isolated (feed-forward) unit (known as central pattern generator) and commanded as joint positions. However, in contrast to the optimization based approaches (mentioned above) and the motivation of the present work, the intrinsic system dynamics is disregarded and a rigid robot design without intrinsic resonance is used. This motivates the investigation of alternative approaches.

On the basis of our initial work [11], this paper focuses on the generation of cyclic motions. It is a known fact that un-damped, elastic multi-body systems tend to show chaotic behavior [12], [13]. This motivates us asking under which conditions VIA robotic (and human) limbs can display periodic motions, how easily can they be induced and how robustly can they be stabilized. Simple experiments with passive systems (Fig. 1) suggest that humans can easily induce such nonlinear oscillations. Despite the complexity of the associated optimal control problem, humans seem to be able to excite independent nonlinear oscillatory modes of the system without difficulty.

The above hypothesis is verified by means of hardware in the loop simulations, where a human controls a realtime simulation of a compliantly actuated arm using a force feedback device. The visco-elastic parameters of the joints are varied within consecutive trials to evaluate their influence on the limit cycles. An important finding of the experiments is that the existence of easily excitable cyclic motions is predominantly determined by the damping properties of the system. A damping analysis of the eigenmodes, based on instantaneous values of the inertia, stiffness, and damping matrix at the equilibrium point, already allows to predict whether the intrinsic system behavior tends to first mode cyclic motions or not, although the open-loop system is strongly nonlinear. If the modal damping is such that oscillations excited in the other modes decay faster than oscillations of the first mode, a simple multi-step bang-bang feedback controller achieves coordinated cyclic motions.

Here, we extend our previous work [11]: since this study 


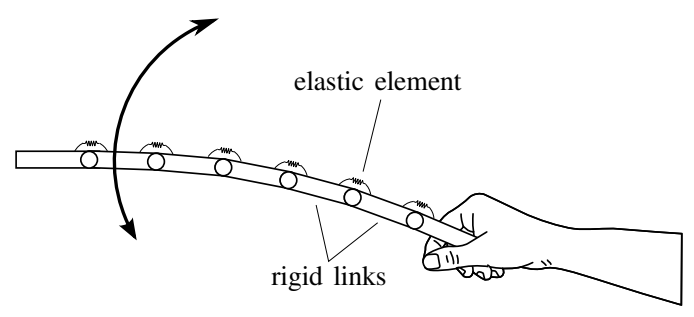

Fig. 1. Human induces cyclic movements for a rod consisting of rigid links, which are connected via nonlinear, elastic elements.

is strongly motivated by biomechanics of humans, the above hypothesis is verified also for the human arm dynamics. Therefore, mechanical properties of the human arm, previously identified in [14], are used within simulations. Similar identification work has been reported in [15], [16], [17], [18] and [19]. In contrast to [15]-[19], our method [14] takes the numerical stability of the identification procedure and the closed-loop behavior of the human arm dynamics (i.e. reflexes) into account. In this study, we apply the method presented in [14] and interpret the results in a modal representation. The analysis of the measurements from nonlinear oscillation perspective confirms that modal damping of the human arm is such that it can easily exhibit first mode cyclic motions. Finally, we verify the approach on a real VIA system. This way, we close the loop from hypotheses to verification using simulations, human data, and robotic experiments. Although, in this paper, the investigations are done for arms because of the availability of arm hardware and human arm models, the results are of course equally valid for legs and other serially structured elastic multi-body systems.

This paper is organized as follows: First, the considered robotic system is introduced and the model nonlinearities are emphasized. Then, the problem is stated in Section II and main hypotheses are posed in Section III. Furthermore a simple bang-bang controller is proposed based on the analysis of the human behaviour. To validate our hypotheses in experiments, three main steps are performed (see Fig. 2): In Section IV qualitative system requirements for multi degree of freedom oscillations are deduced from experiments, where a human controls a robotic arm. In Section V and VI the case where the bang-bang controller induces oscillations in a robotic arm is considered and the influence of modal parameters on nonlinear oscillations is analyzed by both, simulation and experiment. Section VII covers the case where the controller stabilizes cyclic motions of a human arm simulation. The presented approach is introduced for fixed stiffness presets. Therefore, Section VIII concludes the work and looks out on possible exploitations of the stiffness variability.

\section{Problem statement}

This paper aims to understand periodic motions of compliantly actuated systems for controlling robots. In this section we state the problem: First, we introduce the model of the compliantly actuated multi-joint system. Second, we consider

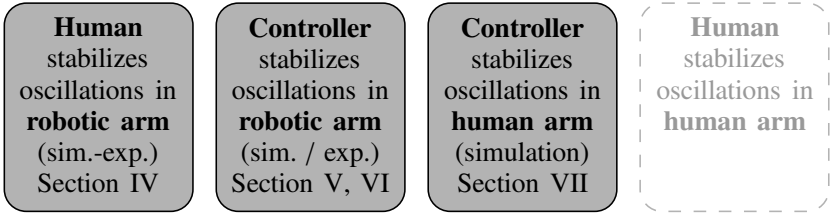

Fig. 2. Main contributions of this paper: constellations considered for the analysis of nonlinear oscillations in human and robotic arms.

a single joint and discuss the difference between a linear and a nonlinear spring characteristic. Finally, we briefly discuss the additional difficulties due to the multi degree of freedom structure.

\section{A. Modeling assumptions}

We consider multi-joint VIA systems that can be generally modeled by Euler-Lagrange equations [20], [21], satisfying:

$$
\frac{\mathrm{d}}{\mathrm{d} t}\left(\frac{\partial L(\boldsymbol{x}, \dot{\boldsymbol{x}})}{\partial \dot{\boldsymbol{x}}}\right)-\frac{\partial L(\boldsymbol{x}, \dot{\boldsymbol{x}})}{\partial \boldsymbol{x}}=\tau_{\mathrm{gen}}+\tau_{\mathrm{dis}} .
$$

The Lagrangian $L(\boldsymbol{x}, \dot{\boldsymbol{x}})=T(\boldsymbol{x}, \dot{\boldsymbol{x}})-U(\boldsymbol{x})$ comprises the kinetic energy $T(\boldsymbol{x}, \dot{\boldsymbol{x}})$ and potential energy $U(\boldsymbol{x})$. Due to the design of VIA systems, not all of the system states $\boldsymbol{x}, \dot{\boldsymbol{x}} \in \mathbb{R}^{m+n}$ are directly actuated. Therefore, let us partition the generalized coordinates $\boldsymbol{x}=\left(\boldsymbol{\theta}^{T}, \boldsymbol{q}^{T}\right)^{T}$ as $\boldsymbol{\theta} \in \mathbb{R}^{m}$ being directly actuated states (motor positions) and $\boldsymbol{q} \in \mathbb{R}^{n}$ being indirectly actuated states (link positions). Accordingly, the generalized force $\tau_{\text {gen }}=\left(\tau_{\mathrm{m}}^{T}, \tau_{\text {ext }}^{T}\right)^{T}$ is composed of the control input $\tau_{\mathrm{m}}$ and externally applied torques $\tau_{\text {ext }}$. Furthermore, $\tau_{\text {dis }}$ represents a generalized, dissipative force, satisfying $\dot{\boldsymbol{x}}^{T} \tau_{\mathrm{dis}} \leq 0$.

In the following, we consider the motor PD control

$$
\boldsymbol{\tau}_{\mathrm{m}}=-\boldsymbol{K}_{D} \dot{\boldsymbol{\theta}}-\boldsymbol{K}_{P}\left(\boldsymbol{\theta}-\boldsymbol{\theta}_{d}\right),
$$

with symmetric and positive definite gains $\boldsymbol{K}_{D}, \boldsymbol{K}_{P} \in \mathbb{R}^{m \times m}$, and suppose that the controlled plant fulfills the following assumptions:

- The coupling inertias in between motor and link side can be neglected ${ }^{1}$.

- The motor side dynamics are sufficiently fast to be neglected $^{2}$.

These simplifying assumptions are fully justified, for instance, for the DLR Hand Arm System [25] and yield dynamic equations of the form:

$$
\boldsymbol{M}(\boldsymbol{q}) \ddot{\boldsymbol{q}}+(\boldsymbol{C}(\boldsymbol{q}, \dot{\boldsymbol{q}})+\boldsymbol{D}) \dot{\boldsymbol{q}}+\frac{\partial U(\boldsymbol{\theta}, \boldsymbol{q})}{\partial \boldsymbol{q}}=\mathbf{0}
$$

The matrices $\boldsymbol{M}(\boldsymbol{q})$ (symmetric and positive definite) and $\boldsymbol{C}(\boldsymbol{q}, \dot{\boldsymbol{q}})$ represent inertia and Coriolis/centrifugal matrix of the rigid-body dynamics, respectively, and $\boldsymbol{D}$ denotes a symmetric and positive definite damping matrix. Considering the above assumptions, we define the motor position $\boldsymbol{\theta}$ as control input, i.e. $\boldsymbol{u}:=\boldsymbol{\theta} \approx \boldsymbol{\theta}_{d}$. The potential energy $U(\boldsymbol{\theta}, \boldsymbol{q})=U_{g}(\boldsymbol{q})+$ $U_{\psi}(\boldsymbol{\theta}, \boldsymbol{q})$ is in general composed of the gravity potential $U_{g}(\boldsymbol{q})$ and the elastic potential $U_{\psi}(\boldsymbol{\theta}, \boldsymbol{q})$. Throughout the rest of

\footnotetext{
${ }^{1}$ This assumption is fulfilled in the presence of high gear ratios, cf. [22].

${ }^{2}$ Singular perturbation assumption, cf. e.g. [23], [24].
} 


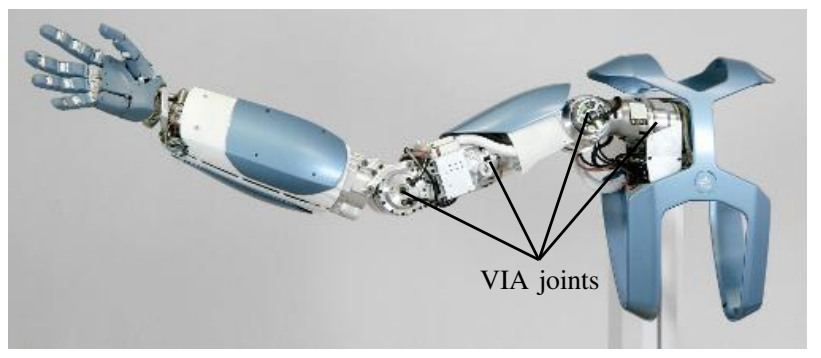

(a) The DLR Hand Arm System

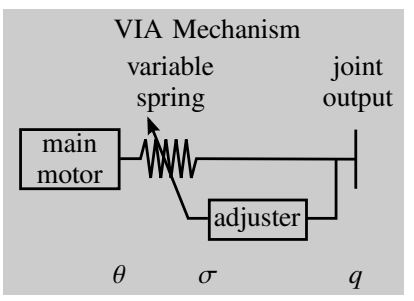

(b) VIA mechanism

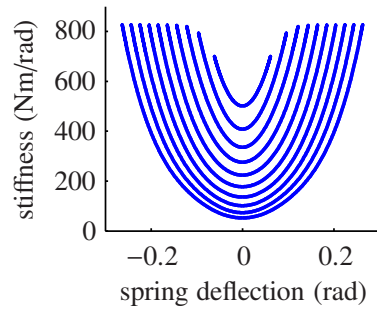

(c) Stiffness of the VIA mechanism
Fig. 3. Description of the arm of the DLR Hand Arm System. Fig. 3(a) highlights the VIA joints implemented as sketched in Fig. 3(b). Fig. 3(c) depicts the stiffness characteristic for adjuster positions $\sigma=\{0,0.02, \ldots, 0.18\}$. Herein, the most outer curve corresponds to $\sigma=0$.

this work, we change the spring characteristic only statically. Therefore, we consider the case $m=n$ and introduce the parameters $\sigma \in \mathbb{R}^{m}$ that change the characteristic of the deflection-force relation, i.e.

$$
\boldsymbol{\psi}(\boldsymbol{\theta}-\boldsymbol{q}, \boldsymbol{\sigma}):=-\frac{\partial U_{\psi}(\boldsymbol{\theta}, \boldsymbol{q}, \boldsymbol{\sigma})}{\partial \boldsymbol{q}} .
$$

\section{B. The arm of the DLR Hand Arm System}

As an example of a VIA system, we introduce the arm of our prototypical VIA robot DLR Hand Arm System shown in Fig. 3 and comprehensively described in [25]. The arm (excluding the lower arm rotation and the wrist) consists of a 4 DoF kinematics chain. Thereby, each joint is equipped with a VIA mechanism implemented as a main motor in series with a nonlinear spring and a much smaller motor to adjust the stiffness characteristic [26]. The deflection-force relation of the nonlinear, adjustable springs can be approximated by cubic functions of the form:

$$
\psi_{i}\left(\theta_{i}-q_{i}, \sigma_{i}\right)=\alpha_{i}\left(\sigma_{i}\right)\left(\theta_{i}-q_{i}\right)+\beta_{i}\left(\sigma_{i}\right)\left(\theta_{i}-q_{i}\right)^{3} .
$$

This relation results from the mechanical design of the VIA joints, detailed in [26]. The order of nonlinearity introduced due to the mechanically implemented floating spring joint is depicted in Fig. 3(c) for several stiffness presets. In the case of the lowest preset $\sigma=0$ the variation of the stiffness is about $1400 \%$ between minimum and maximum spring deflection. Note that the joint stiffness is changing with the joint deflection.

\section{Single-joint case}

Consider the model

$$
I \ddot{q}+d \dot{q}=\psi(u-q),
$$

of a single compliantly actuated joint. The control input $u \in \mathbb{R}$ (motor position) acts via the spring $\psi(u-q)$ (defined in (5)) on the link inertia $I$, with joint position $q \in \mathbb{R}$. Further, $d$ specifies the viscous damping force.

The objectives of the paper are:

- Deriving a control $u$ such that $q(t)=q(t+\Delta t)$ for a certain $\Delta t$

- Exploiting mechanical resonance effects of the system.

Therefore, we consider a sinusoidal control signal $u(t)$ and compare qualitatively the behavior of a system consisting of a linear spring (i.e., $\alpha>0, \beta=0$ in (5)) and a nonlinear, cubic spring (i.e., $\alpha, \beta>0$ in (5)):

1) Linear spring: The resulting system is a forced, linear oscillator. A resonance oscillation can be reached either by exciting the system with a frequency close to the natural frequency or in case of a VIA joint with a linear and adjustable spring by adjusting the stiffness such that the resulting natural frequency is close to the desired frequency of the task and excitation.

2) Nonlinear, cubic spring: The system represents a forced, nonlinear, parametrically excited oscillator with multifrequency excitation. Approximative solutions can be obtained partly using perturbation methods [27], [28]. The qualitative behavior is discussed in [12]:

- Cubic nonlinearity: the system exhibits multiple resonances; The amplitude and frequency of the steady-state response depend on the excitation (amplitude, frequency) and the initial conditions.

- Parametric excitation: the system consists of rapidly varying parameters; small excitation amplitudes can produce large responses, even if the excitation frequency is not close to the linear, natural frequency.

- Multi-frequency excitation: more than one type of excitation may occur simultaneously.

These observations highlight that there is a substantial difference, even in the single-joint case, between the well known linear case and the nonlinear case, making the prediction of cyclic motions non-trivial.

\section{Multi-joint case}

This paper aims to investigate multi-joint periodic motions, i.e. trajectories for which $q(t)=\boldsymbol{q}(t+\Delta t)$, for a certain $\Delta t$. Thereby, the goal is to derive a control $\boldsymbol{u}$ that exploits the elastic energy storage $U_{\psi}(\boldsymbol{u}, \boldsymbol{q})$ of systems, satisfying (3). In our previous work [29], we achieved the above objectives by slightly changing (by feedback linearization) the original dynamics of the plant. In this work, we aim to avoid changing the intrinsic dynamical behavior of the plant.

Let us clarify the problem by means of the linear system:

$$
M \ddot{q}+D \dot{q}+K \boldsymbol{q}=\boldsymbol{K u},
$$

where $\boldsymbol{M}, \boldsymbol{D}, \boldsymbol{K} \in \mathbb{R}^{n \times n}$ are constant, symmetric, and positive definite matrices. According to Lemma 1 given in Appendix A, the control law

$$
\boldsymbol{u}(t)=\boldsymbol{K}^{-1} \boldsymbol{Q}^{-T}\left[\begin{array}{c}
\hat{u}_{z} \cos \omega_{1} t \\
\mathbf{0}
\end{array}\right],
$$


achieves a resonance excitation along the first eigenmode of the linear system (7) in a well known way.

In contrast, the system (3) considered in this work is strongly nonlinear. Therefore, a resonance-like excitation similar to (8) would require to extend the notion of linear eigenmodes to so-called nonlinear normal modes [30]. In the particular case of (3), the method proposed in [30] requires to solve a system of nonlinear partial differential equations in $2 n-2$ dependent variables. This seems unfeasible in our case, since an analytic solution does not exist in general [30]. In the following, we are looking to the strategies used by humans exiting oscillations and derive a simple control law based on these observations.

\section{Controller DESIGN BASED ON CONTROL STRATEGIES OF HUMANS}

In this section, based upon human experiments we derive a control law to stabilize periodic motions for compliantly actuated systems (3) introduced in Section II-A. Despite the current theoretical difficulties discussed in Section II-C, II-D, humans are able to stabilize periodic motions easily, even in the presence of strong nonlinearities and multiple degrees of freedom. This has been confirmed by simple experiments, where a human induces oscillations into a rod (see, Fig. 1). Stable oscillations could be achieved even for the case of large deflections (i.e. in the presence of strong nonlinearities). The human does not need a long training phase to do so. This demonstrates the ability of humans to control periodic motions of nonlinear multi degree of freedom systems. From these observations we hypothesize that:

- The motor control of humans is able to stabilize periodic motions even in the presence of strong nonlinearities.

- The underlying control law has a simple and very robust structure.

On the basis of experimental observations from strategies used by humans, a control law will be derived that confirms these hypotheses.

Accessing and measuring control and feedback signals of humans during natural motions is difficult and largely unresolved [31]. We circumvent this problem by using hardware in the loop simulations with human control. Using a force feedback device, a human operator can be coupled in the feedback control loop with either a robotic plant or a simulated system. The latter allows to adjust the system parameters arbitrarily as done in the following experiments.

\section{A. Experimental setup and procedure}

To include the force feedback device in the control loop of the system (3), the $k$-th motor position of the robot is considered as control input, i.e., $u:=\theta_{i}$ if $i=k$, otherwise $\theta_{i}=$ const. The spring torque (cf. (4)) of the same joint is considered as system output. With states $\boldsymbol{x}=\left(\boldsymbol{q}^{T}, \dot{\boldsymbol{q}}^{T}\right)^{T}$, the system (3) turns into the single-input single-output system:

$$
\begin{aligned}
& \dot{\boldsymbol{x}}=\boldsymbol{f}(\boldsymbol{x}, u)=\left[-\boldsymbol{M}(\boldsymbol{q})^{-1}\left(\frac{\partial U(\boldsymbol{\theta}, \boldsymbol{q})}{\partial \boldsymbol{q}}+(\boldsymbol{C}(\boldsymbol{q}, \dot{\boldsymbol{q}})+\boldsymbol{D}) \dot{\boldsymbol{q}}\right)\right], \\
& y=\psi_{k}\left(u-q_{k}\right) .
\end{aligned}
$$

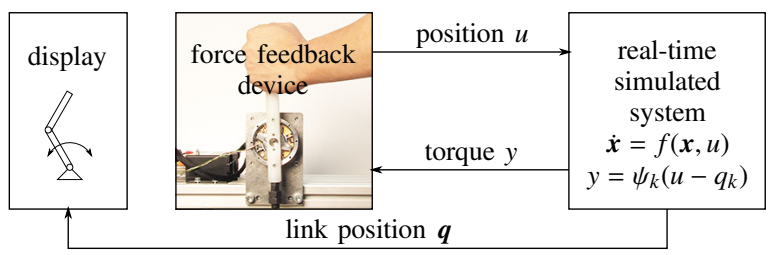

Fig. 4. Experimental setup to include a human in the control loop of a realtime simulated VIA system. Haptic feedback is provided by a force feedback device. Visual feedback is given by a display showing a real-time simulation of a robot.

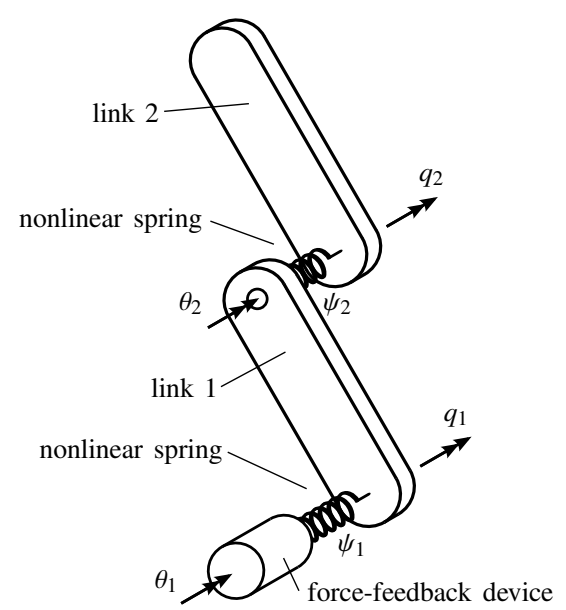

Fig. 5. Technical scheme of the hardware in the loop simulation. The complete system: double pendulum including nonlinear visco elasticities is simulated in real time. Position of the feedback device is the control input of the simulated system, i.e., $u:=\theta_{1}$ and $\theta_{2}=$ const.. The spring torque (of the first joint) $\psi_{1}$ acts as force feedback.

As sketched in Fig. 4, the real time simulation of (9) and (10) was interconnected with a direct drive (torque controlled) motor with a handle mounted on the rotor. This motor acts as force feedback device. An optical encoder provides the angular position of the motor as control signal $u:=\theta_{1}$ for the simulated VIA arm (9). The joint torque $y=\psi_{1}\left(u-q_{1}\right)$ computed by the VIA arm simulation is commanded to the current controller of the force feedback device and thereby provides feedback to the human operator. This setup allows to emulate arbitrary dynamic systems that are controlled by a single position input / torque output, and interface them to a human operator.

In a series of experiments, the oscillatory behavior of a planar VIA double pendulum (i.e., $\boldsymbol{q} \in \mathbb{R}^{2}, \boldsymbol{\theta} \in \mathbb{R}^{2}, u:=\theta_{1}$, and $\theta_{2}=0$ ) was analyzed (see, Fig. 5). The double pendulum has been oriented w.r.t. the gravity vector such that $\partial U_{g}(\boldsymbol{q}) / \partial \boldsymbol{q}=$ 0. Besides inertial dynamics, strong nonlinear cubic springs (see (5)) were considered, where the ratio of linear and cubic spring constants was chosen as $\beta_{i} / \alpha_{i}=70$ (similar to the most nonlinear case of the floating spring mechanism, cf. Fig. 3(c)). To comply with the range of maximum torques of the force feedback device $\tau_{\max }= \pm 1 \mathrm{Nm}$, inertia and spring parameters were adjusted, see Table I. Furthermore, damping parameters $\boldsymbol{D}=\operatorname{diag}(0.008,0.008) \mathrm{Nms} / \mathrm{rad}$ have been considered. The system (9) was integrated (forward Euler method, time steps $0.001 \mathrm{~s}$ ) on the same real time computer on which the force feedback device was controlled. Additionally, the motion of 
TABLE I

SySteM PARAMETERS FOR THE HARDWARE IN THE LOOP SIMULATION

\begin{tabular}{|c||c||c||c||c|}
\hline $\mathrm{i}$ & mass $m_{i}$ & link length $l_{i}$ & center of mass $l_{c i}$ & linear spring $\alpha_{i}$ \\
\hline 1 & $0.6 \mathrm{~kg}$ & $0.1 \mathrm{~m}$ & $0.05 \mathrm{~m}$ & $0.03 \mathrm{Nm}$ \\
\hline 2 & $0.6 \mathrm{~kg}$ & $0.1 \mathrm{~m}$ & $0.05 \mathrm{~m}$ & $0.03 \mathrm{Nm}$ \\
\hline
\end{tabular}

the double pendulum was visualized on a screen.

One skilled participant ${ }^{3}$ was tested. To initialize the tests, the subject grasped the handle of the force feedback device and rested in a centred, initial position (cf. Fig. 4), while the integrator was reset. Then the subject moved the handle to induce oscillations. The goal was to achieve and stabilize coordinated, cyclic movements.

\section{B. Experimental results: derivation of the control law}

Fig. 6 shows measurements of the motor position $\theta_{1}(t)$ (control signal), spring torque $\psi_{1}(t)$ (feedback signal), and link position $q_{1}(t)$ corresponding to the first joint. The oscillation of $\theta_{1}(t)$ and $\psi_{1}(t)$ is synchronized, while the motion of $q_{1}(t)$ is shifted by half an oscillation period. At a certain level of the spring torque $\psi_{1}$, the motor position $\theta_{1}$ reaches approximately a plateau, which lasts as long as the spring torque $\psi_{1}$ undershoots another level. These observations indicate that the human tries to synchronize the energy input to the torque respectively link position peaks. When a certain spring deflection (torque) is detected, the human countered it by moving the motor (control signal) in the opposite direction of the link deflection and thereby inducing energy into the system. Such a behavior can be approximately replicated by the discontinuous control law

$$
\theta_{d}(\Delta \tau)=\theta_{\mathrm{e}, 1}+\left\{\begin{array}{ll}
\operatorname{sign}(\Delta \tau)|\hat{\theta}| & \text { if }|\Delta \tau|>\epsilon_{\tau} \\
0 & \text { otherwise }
\end{array} .\right.
$$

This multi step bang-bang controller is triggered by the deviation of the spring torque $\psi_{1}\left(\theta_{1}-q_{1}\right)$ w.r.t. the equilibrium torque $\psi_{\mathrm{e}, 1}\left(\theta_{\mathrm{e}, 1}-q_{\mathrm{e}, 1}\right)$, i.e. $\Delta \tau=\psi_{1}\left(\theta_{1}-q_{1}\right)-\psi_{\mathrm{e}, 1}\left(\theta_{\mathrm{e}, 1}-q_{\mathrm{e}, 1}\right)$, where the equilibrium positions $\boldsymbol{\theta}_{\mathrm{e}}$ and $\boldsymbol{q}_{\mathrm{e}}$ satisfy

$$
\left.\frac{\partial U(\boldsymbol{\theta}, \boldsymbol{q})}{\partial \boldsymbol{q}}\right|_{\boldsymbol{\theta}=\boldsymbol{\theta}_{\mathrm{e}}, \boldsymbol{q}=\boldsymbol{q}_{\mathrm{e}}}=\mathbf{0} .
$$

Note that the system considered in this section fulfills condition (12) if $\boldsymbol{\theta}_{\mathrm{e}}=\boldsymbol{q}_{\mathrm{e}}$. Therefore, $\boldsymbol{\psi}_{\mathrm{e}}=\mathbf{0}$. Fig. 7 shows the action principle of the controller (11) connected exemplary to the same system as considered in the experiments. When the torque $\Delta \tau=\psi_{1}$ exceeds a certain threshold $\epsilon_{\tau}$, the controller induces a fixed amount of energy into the system. This is achieved by a step $\hat{\theta}$ in the motor position w.r.t the initial position $\theta_{\mathrm{e}}$. Thereby, the step like elongation is in the opposite direction of the current link deflection $q_{1}$.

\section{Comments on the control law}

The control signal generated by the human operator is continuous (see, Fig. 6). In contrast, the output of the bangbang controller is discontinuous (see, Fig. 7). The step-like

\footnotetext{
${ }^{3}$ The participant was involved in the experimental background.
}

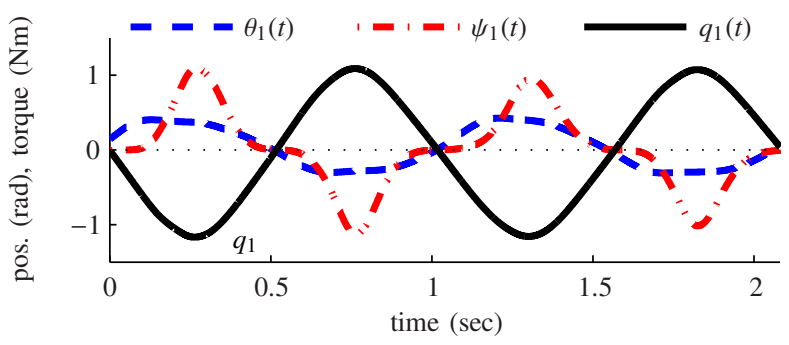

Fig. 6. Two periods of motion recorded in the steady-state phase of the oscillation experiment, where a human operator controls a real time simulated VIA double pendulum. The progress of the motor position $\theta_{1}(t)$ (control action of the human), spring torque $\psi_{1}(t)$ (feedback signal), and the link position $q_{1}(t)$ is depicted. For clarity of presentation the second link position is omitted.

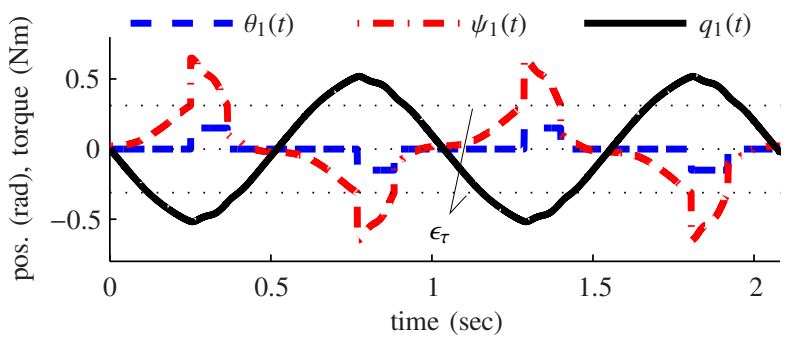

Fig. 7. Action principle of the multi-step bang-bang controller derived from experimental observations. The controller is connected to the same system as considered in the experiments where a human operator is in the control loop (cf. Fig. 6). The controller parameters are adjusted such that the frequency of the link motion is similar to the motion plotted in Fig. 6.

excitation is required, since the controller lacks the knowledge of the intrinsic oscillation frequency of the plant. Therefore, the controller parameters threshold $\epsilon_{\tau}$ and switching amplitude $\hat{\theta}$ can be adjusted such that the resulting oscillation is either close to the intrinsic, oscillatory behavior of the plant or fits (in a certain range) the desired frequency of the considered task. Furthermore, it should be noted that the original control signal of the human operator acts on the muscles and not directly on the handle of the feedback device. That means that there are filter dynamics between the original control signals and the signal measured during the experiment (motor position). Therefore, the original control signal might be discontinuous, even if the motor position is continuous.

\section{ExPERIMENTAL StUdY: INFLUENCE OF THE DAMPING ON HUMAN CONTROLLED OSCILLATIONS}

The experiments described in Section III-A revealed that given the considered setup, it is straightforward for a human operator to stabilize multi-degree of freedom cyclic movements. Even more, the system tends to show only first mode motions, where the motion of the first and second link is periodic and in phase. Preliminary experiments indicate that it might be a result of the damping distribution in the considered plant. For equal and constant joint damping, the modal analysis of the linearized system (at the equilibrium point) revealed that the first eigenmode is less damped than the second one. (Definitions 1 and 3 of the linearized system respectively the modal damping are given in the Appendix A.) We hypothesize that the existence of easily excitable oscillation modes depends on the distribution of the modal damping. Therefore, the objective 


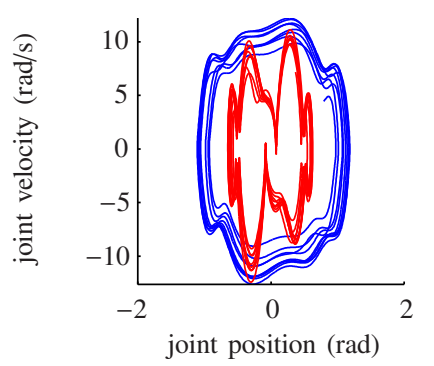

(a) $\xi=0.1,1.0$

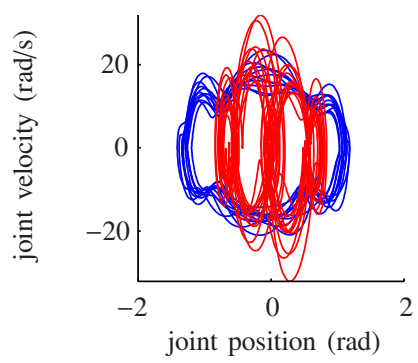

(e) $\xi=0.1,0.5$

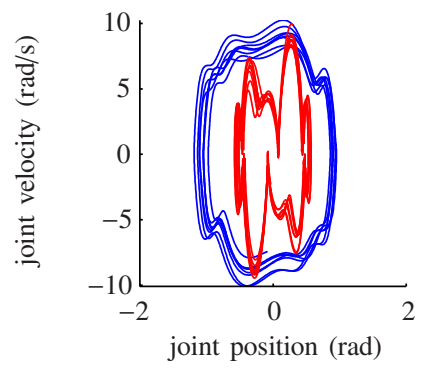

(b) $\xi=0.1,0.9$

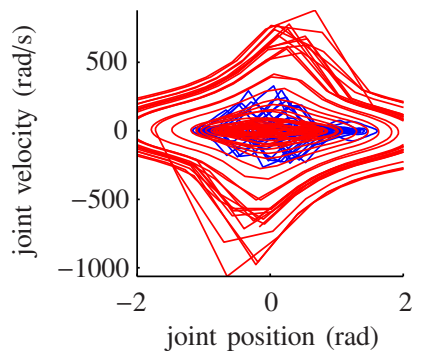

(f) $\xi=0.1,0.4$

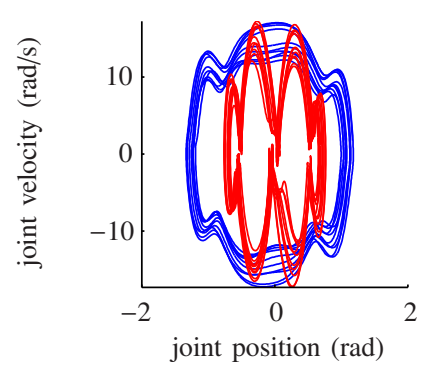

(c) $\xi=0.1,0.8$

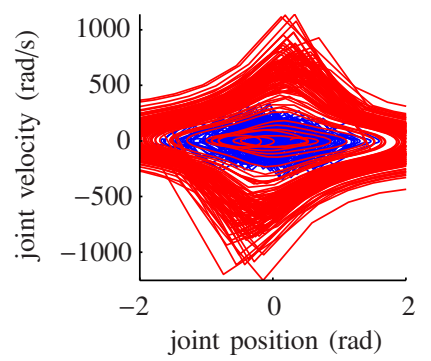

(g) $\xi=0.1,0.2$

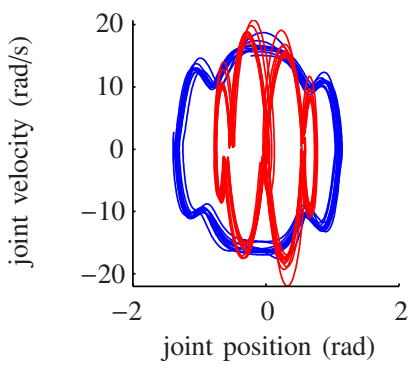

(d) $\xi=0.1,0.6$

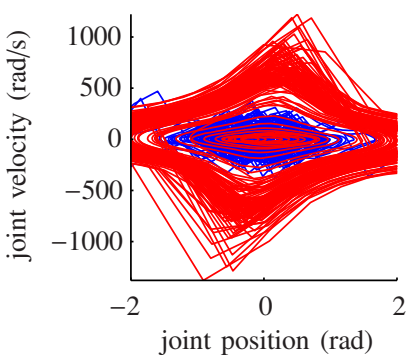

(h) $\xi=0.1,0.1$

Fig. 8. Phase plots of human controlled oscillations. Blue lines represent the pair $\left(q_{1}, \dot{q}_{1}\right)$ and red lines the pair $\left(q_{2}, \dot{q}_{2}\right)$. The plotted data is recorded after a run-in period of approximately $8 \mathrm{sec}$. The eigenfrequecies $\omega_{1}(\mathbf{0})=1.9(\mathrm{rad} / \mathrm{s})$ and $\omega_{2}(\mathbf{0})=20.9(\mathrm{rad} / \mathrm{s})$ were constant over all trials, while the modal damping factors $\boldsymbol{\xi}(\mathbf{0})$ had been varied.

of the following experiments is validating this hypothesis by directed tests.

\section{A. Experimental procedure}

The same experimental setup and procedure as described in Section III-A, were considered. Except the stiffness and damping parameters, the parameters of the real-time simulated system were equal. The stiffness parameters were $\alpha_{1}=$ $0.02 \mathrm{Nm} / \mathrm{rad}, \alpha_{2}=0.01 \mathrm{Nm} / \mathrm{rad}$ and the ratio for the cubic coefficients remained $\beta_{i} / \alpha_{i}=70$. Modal damping $\xi_{i}$ of the linearized system (at the equilibrium point) was adjusted such that $\boldsymbol{D}=$ const. during each trial using Definition 4 given in the Appendix A. The damping of the first mode was held low and constant, while the damping of the second mode has been varied between trials.

\section{B. Experimental results}

Phase plots of the joint motion $\boldsymbol{q}(t)$ vs. $\dot{\boldsymbol{q}}(t)$ are displayed in Fig. 8.

1) The influence of modal damping: Nonlinear effectsinduced by inertia couplings, Coriolis/centrifugal effects, and the progressive stiffness characteristic of the springs-increase when the damping factor of the second mode converges to the one of the first mode. These effects are expressed in form of strong notches towards the center in the shape of circular or elliptical paths. Severe changes occur when the damping of the second mode falls below approximately 0.5 ; then bifurcation arises and the system tends to non-periodic behavior, causing numerical instabilities of the simulation (cf. 8(f)-(h)).
2) The steady-state of cyclic motions: For ideal cyclic motions a phase plot trajectory of one position-velocity pair is a single closed path. For trajectories depicted in Fig. 8, the motion of each joint is at most bounded by an inner and outer closed path (and not a single closed path). The reason therefore can be limitations in the range and sensitivity of feedback signals given to the human operator. Additionally, the control signals generated by humans may not be sufficiently accurate and repeatable. The deterministic controller derived in Section III-B allows to avoid these uncertainties.

\section{Simulation Study: Influence of The STIFFness AND DAMPING ON MULTI-STEP BANG-BANG CONTROLLED OSCILLATIONS}

In the last section it was demonstrated that even in the presence of strong nonlinearities, multi degree of freedom cyclic movements can be induced easily by a human. Now, we investigate intrinsic system properties, which ensure that the system tends to periodic motions. The goal of this section and the following Sections III-A and VII is showing whether the proposed controller is able to excite and sustain periodic motions in serial type elastic systems as appearing in robotics and biology. The following study will provide a qualitative validation for human and robotic arms and possibly legs. Thereby, the interpretation of the results will require to clarify some nomenclature.

\section{A. Nomenclature: oscillation modes}

We consider the notion of oscillation modes for $2 n$-th order nonlinear systems introduced in [30]. Therefore, it is assumed that the motion in the state space $(\boldsymbol{q}, \dot{\boldsymbol{q}}) \in \mathbb{R}^{2 n}$ can be expressed 
in terms of an arbitrary single coordinate-velocity pair $(v, w) \in$ $\mathbb{R}^{2}$ such that

$$
\left(\begin{array}{c}
\boldsymbol{q} \\
\dot{\boldsymbol{q}}
\end{array}\right)=\boldsymbol{g}(v, w) \in \mathbb{R}^{2 n}
$$

Choosing, for instance, $v=q_{1}$ and $w=\dot{q}_{1}$, the $2 n-2$ remaining constraints in (13) define a surface of dimension 2 . The motion that takes place on this 2-dimensional surface (and satisfies the differential equation of the considered system) is called an oscillation (or normal) mode. In general, seeking functions $\boldsymbol{g}(v, w)$ leads to a system of nonlinear partial differential equations, where exact solutions cannot be computed. However, it can be shown that in a neighborhood of an equilibrium point there exist $n$ solutions $\boldsymbol{g}_{i}(v, w)$, each corresponding to a mode of the considered system [30]. On the basis of these considerations, we propose the following nomenclature:

- If the trajectory $\dot{\boldsymbol{q}}(t)$ vs. $\boldsymbol{q}(t)$ represents a closed path in the state space, we denote this motion an oscillation mode of the system. In that case, evidently, the projections of the motion for each joint $j$ onto the plane (i.e. $\dot{q}_{j}(t)$ vs. $\left.q_{j}(t)\right)$ are periodic.

- The period of one cycle determines the frequency of the oscillation mode. If for one cycle each of the projected joint motions is unique (the closed paths do not intersect themselves), we refer to this motion as a first mode motion ${ }^{4}$.

- If for one cycle at least one of the projected joint motions is not unique, we refer to this motion as a first mode motion, where additional modes are excited ${ }^{5}$. In that case, multiple oscillation frequencies are present. Note that if more than one oscillation mode is excited, either the frequencies have to be multiples of each other, or the oscillation of the faster (higher frequency) modes have to decay sufficiently fast, otherwise the resulting motion becomes non-periodic.

Throughout, we use the terminologies of eigenfrequency and modal damping. Thereby, we refer to quantities of the linearized system (at the equilibrium point) defined in the Appendix A. It should be emphasized that these quantities are only used as points of reference to qualitatively discuss the behavior of the nonlinear systems and are not intended to explain the results.

\section{B. Simulation details}

Again, as in Section III and IV, the system considered is the compliantly actuated double pendulum of the form (9) with cubic springs defined by (5) in the joints, where the motor position $u:=\theta_{1}$ of the first joint acts as control input and the elastic torque $\psi_{1}\left(u-q_{1}\right)$ is used as feedback. The remaining

\footnotetext{
${ }^{4}$ Note that if each of the projected joint motions is periodic and unique, it is possible to parametrize the $2 n$-dimensional motion in terms of the coordinatevelocity pair of any joint according to (13).

${ }^{5}$ If one of the projected joint motions is not unique (for one cylce), the corresponding coordinate-velocity pair cannot serve as parametrization of the $2 n$-dimensional motion. Since in that case, the choice of the coordinatevelocity pair is not arbitrary, this contradicts the definition of an oscillation mode in (13). Therefore, at least an additional coordinate-velocity pair must be considered to describe the $2 n$-dimensional motion.
}

TABLE II

Inertial parameters of THE DLR HaNd Arm System

\begin{tabular}{|c||c||c||c|}
\hline $\mathrm{i}$ & mass $m_{i}$ & link length $l_{i}$ & center of mass $l_{c i}$ \\
\hline 1 & $5.0 \mathrm{~kg}$ & $0.4 \mathrm{~m}$ & $0.2 \mathrm{~m}$ \\
\hline 2 & $5.0 \mathrm{~kg}$ & $0.4 \mathrm{~m}$ & $0.2 \mathrm{~m}$ \\
\hline
\end{tabular}

motor position $\theta_{2}=0$ is hold constant. To obtain repeatable results, oscillations are induced by means of the control law (11) derived in the last section instead of the human operator. The controller parameters are set to $\hat{\theta}=0.3 \mathrm{rad}$ and $\epsilon_{\tau}=30 \mathrm{Nm}$ for all simulations. Although the considered system is nonlinear, the stiffness and damping parameters will be adjusted based on modal analysis of the linearized system (at the equilibrium point) where $\alpha_{i}=$ const., $\beta_{i}=$ const., and $\boldsymbol{D}=$ const. during each simulation run. For the link side mass matrix at zero position $\boldsymbol{M ( 0 )}$ fixed, desired eigenfrequencies are assigned (given below for specific cases), in order to compute the linear spring coefficients $\alpha_{i}$ (see, Problem 1 and Definiton 5 in Appendix A). Then the physical damping matrix $\boldsymbol{D}$ is computed based on the linearized stiffness matrix and given modal damping factors (see, Definition 4 in Appendix A). Finally, coefficients of cubic spring terms are chosen such that $\beta_{1} / \alpha_{1}=\beta_{2} / \alpha_{2}=70$.

All simulations were performed in Matlab/Simulink ${ }^{\circledR}$. The differential equations were integrated by means of the variable step solver ode23t for moderately stiff problems with a maximum step size of $0.0005 \mathrm{sec}$. Initial conditions were set $(0.6,0)$ for joint angles and $(0,0)$ for joint velocities.

\section{Limit cases of modal properties}

Based on the inertial properties of the DLR Hand Arm System given in Table II, two substantially different cases of eigenfrequency distributions are considered:

1) Different eigenfrequencies: As in the case of the DLR Hand Arm System and most VIA robots, mono-articulation is assumed, i.e. no coupling springs between multiple joints are present. As such, a displacement in direction of one joint generates solely a reaction force in the opposite direction of the same joint:

$$
-\frac{\partial U_{\psi}(\boldsymbol{\theta}, \boldsymbol{q})}{\partial q_{i}}=\psi_{i}\left(\boldsymbol{\theta}, q_{i}\right) .
$$

Linearizing these elastic force functions leads to a diagonal stiffness matrix:

$$
\boldsymbol{K}(\boldsymbol{\theta}, \boldsymbol{q})=\operatorname{diag}\left(\frac{\partial^{2} U_{\psi}(\boldsymbol{\theta}, \boldsymbol{q})}{\partial q_{i}^{2}}\right) .
$$

Then, as a consequence of the coupled mass matrix (and for $\boldsymbol{q} \in \mathbb{R}^{2}$ ) there exists a minimum ratio of assignable eigenfrequencies $\omega_{2} / \omega_{1}>v_{\min }(\boldsymbol{M})$, which can be realized by a diagonal stiffness matrix (see, (A19) in Appendix A), i.e. for a given mass matrix $\boldsymbol{M}$ and first eigenfrequency $\omega_{1}$ the second eigenfrequency $\omega_{2}$ must be larger than $\omega_{1} v_{\min }(\boldsymbol{M})$, otherwise a coupled stiffness matrix is required. Throughout the rest of this work we refer to the case of different eigenfrequencies if $\omega_{2} / \omega_{1}>v_{\min }(\boldsymbol{M})$. This case was considered for human controlled oscillations described in the last section and will be 


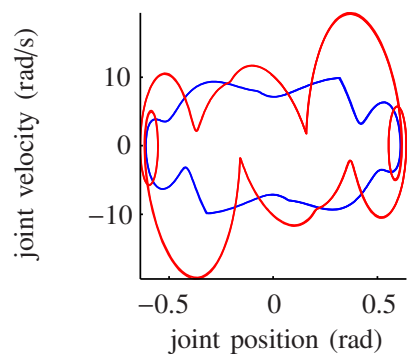

(a) $\xi=0.1,1.0 ; \omega=2.0,21.0$

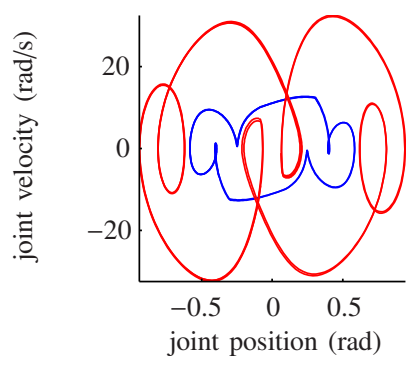

(e) $\xi=0.1,1.0 ; \omega=2.0,5.0$

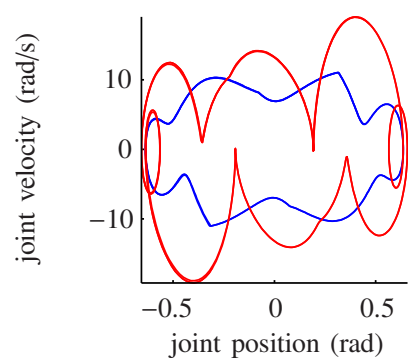

(b) $\xi=0.1,0.7 ; \omega=2.0,21.0$

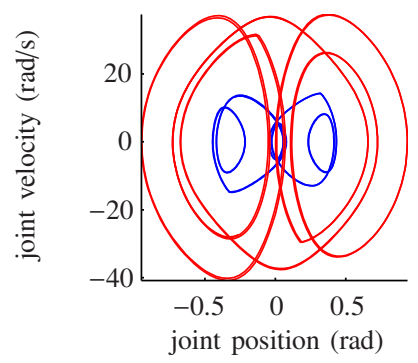

(f) $\xi=0.1,0.7 ; \omega=2.0,5.0$

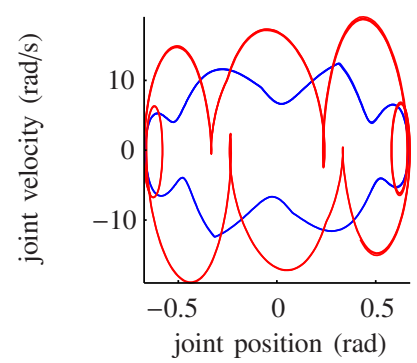

(c) $\xi=0.1,0.4 ; \omega=2.0,21.0$

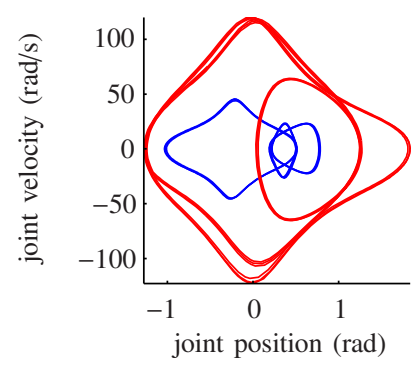

(g) $\xi=0.1,0.4 ; \omega=2.0,5.0$

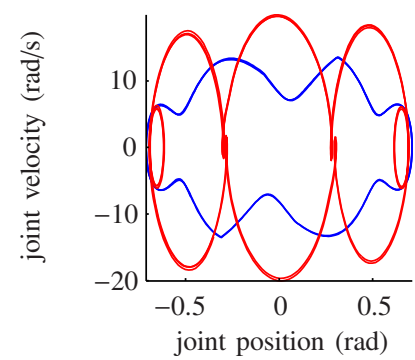

(d) $\xi=0.1,0.1 ; \omega=2.0,21.0$

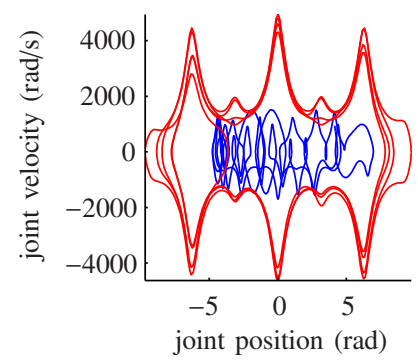

(h) $\xi=0.1,0.1 ; \omega=2.0,5.0$

Fig. 9. Phase plots of simulated motions, where the controller proposed in Section III-B is in the loop. Blue lines represent the pair $\left(q_{1}, \dot{q}_{1}\right)$ and red lines the pair $\left(q_{2}, \dot{q}_{2}\right)$. Simulations displayed correspond to the mass distribution of the DLR Hand Arm System.

analyzed in the following using the deterministic bang-bang controller.

2) Similar eigenfrequencies: In contrast to the case of different eigenfrequencies, we refer to the case of similar eigenfrequencies if the ratio of eigenfrequencies $\omega_{2} / \omega_{1}$ requires to introduce coupling springs, i.e. $\omega_{2} / \omega_{1}<v_{\min }(\boldsymbol{M})$. Coupling springs have the effect that a displacement in one coordinate direction can cause a reaction force in a different coordinate direction:

$$
-\frac{\partial U_{\psi}(\boldsymbol{\theta}, \boldsymbol{q})}{\partial q_{i}}=\psi_{i}(\boldsymbol{\theta}, \boldsymbol{q})
$$

and consequently the stiffness matrix for the instantaneous linearized system contains nonzero off-diagonal entries:

$$
K_{i, j}(\boldsymbol{\theta}, \boldsymbol{q})=\frac{\partial^{2} U_{\psi}(\boldsymbol{\theta}, \boldsymbol{q})}{\partial q_{i} \partial q_{j}} \neq 0 .
$$

Note that nonlinear coupling elasticities are not present in most of today's VIA robot arms, therefore we introduce this artificial case of similar eigenfrequencies here for sake of theoretical insight and comparison with the human arm.

\section{Simulation results}

1) Different eigenfrequencies: Fig. 9(a)-(d) depict phase plots of simulated motions in the case of different eigenfrequencies. For each simulation run eigenfrequencies $\omega_{1}(\mathbf{0})=$ $2 \mathrm{rad} / \mathrm{s}$ and $\omega_{2}(\boldsymbol{0})=21 \mathrm{rad} / \mathrm{s}$ corresponding to $\omega_{2} / \omega_{1}>$ $v_{\min }(\boldsymbol{M})$ were assigned, while the modal damping varies w.r.t. each run. Note that $\omega_{1}$ was chosen arbitrarily and $\omega_{2}$ results due to $v_{\min }(\boldsymbol{M})$. For all presets of modal damping, phase plots of both coordinate directions are closed paths - indicating the tendency of the system to cyclic movements. As in the case of human controlled oscillations the effects of nonlinearities (manifested by strong notches towards the center in the phase plot paths) increase, when the value of the second mode damping converges to the value of the first mode damping. But in contrast to human controlled oscillations (see, Fig. 8(j)) the bang-bang regulator (11) avoids non-periodic, numerically unstable behavior-even in the case of equal and low modal damping (cf. Fig. 9(d)).

To demonstrate the strong occurrence of nonlinearities, time series of the control input $\theta_{1}(t)$, joint angles $\boldsymbol{q}(t)$, joint velocities $\dot{\boldsymbol{q}}(t)$, as well as instantaneous values of the modal damping $\xi(t)$, eigenfrequencies $\omega(t)$, and potential / kinetic energy $U(t)$ / $T(t)$ corresponding to the phase plot Fig. 9(a) are depicted in Fig. 10. It can be observed that only in the equilibrium point $\boldsymbol{q}=\boldsymbol{\theta}=\mathbf{0}$ eigenfrequencies and modal damping equal the assigned values. At these points the modal damping has its maximum and the eigenfrequency its minimum. For increasing magnitudes of spring deflections $\left|q_{i}-\theta_{i}\right|$ both instantaneously linearized eigenfrequencies increase and the modal damping factors decrease. When spring deflections are maximal, the eigenfrequencies / modal damping factors approach their maxima / minima. Additionally, one can identify points where the Hamiltonian energy is almost completely kinetic. This is a typical property for coordinated cyclic movements [32].

2) Similar eigenfrequencies: In the following, we treat the case of similar eigenfrequencies corresponding to the linearized system (at the equilibrium point). Therefore, we consider the mass matrix $\boldsymbol{M ( 0 )}$ as given and tune the entries of the stiffness matrix

$$
\boldsymbol{K}_{0}=\left[\begin{array}{cc}
\alpha_{1}+\alpha_{3} & -\alpha_{3} \\
-\alpha_{3} & \alpha_{2}+\alpha_{3}
\end{array}\right] \text {. }
$$




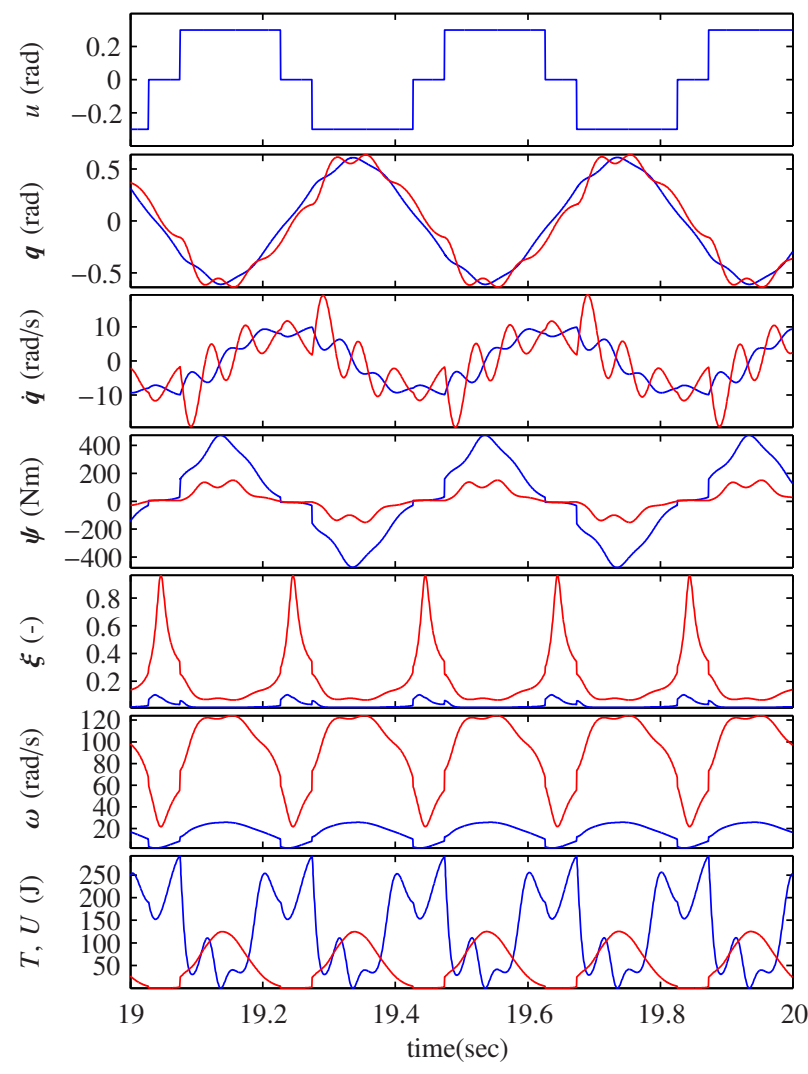

Fig. 10. Time series corresponding to the simulation plotted in Fig. 9(a). Herein $u:=\theta_{1}$ is the motor position of the first joint, i.e. the control action. Link positions, link velocities, and spring torques corresponding to the first (blue) and second (red) joint, are denoted by $\boldsymbol{q}, \dot{\boldsymbol{q}}$, and $\boldsymbol{\tau}$, respectively. Instantaneous values of damping and eigenfrequency corresponding to the first (blue) and second (red) mode of the linearized system (cf. (A2) are denoted by $\xi$ and $\omega$, respectively. The kinetic energy $T$ (blue) and potential energy $U$ (red) of the system are depicted in the last plot.

The resulting entries of the above stiffness matrix correspond to the linear coefficients $\alpha_{i}$ of the springs. In more detail, first, the condition $\omega_{2} / \omega_{1}<v_{\min }(\boldsymbol{M})$ is tested. Then it is decided if the eigenfrequencies can be achieved by a diagonal or coupled stiffness matrix ${ }^{6}$. For each case (diagonal or coupled stiffness matrix) there exist an analytical relation to determine $\alpha_{i}$. The resulting potential function (A10) as well as the force and stiffness functions are given in the Appendix A. Although we adjust the linear spring coefficients $\alpha_{i}$ based on linearization (in the equilibrium point), we consider the nonlinear joint elasticities in simulation.

In simulations the procedure given in the Appendix $\mathrm{A}$ is applied to assign $\omega_{1}=2 \mathrm{rad} / \mathrm{s}, \omega_{2}=5 \mathrm{rad} / \mathrm{s}$, which correspond to the case of similar eigenfrequencies. Therefore, the value of the cubic spring coefficient is chosen $\beta_{3} / \alpha_{3}=70 / 4$. Phase plots of simulated motions for the same damping adjustments as used above are depicted in Fig. 9(e)-(h). It can be observed that even for the case in Fig. 9(e) where the first mode is weakly and the second mode is strongly damped, in addition to first mode motions, the second mode is excited (see, considerations in Section V-A). This is a result of modal coupling

\footnotetext{
${ }^{6}$ Note that for a coupled mass matrix the stiffness matrix has to be also coupled and even $\boldsymbol{K} \propto \boldsymbol{M}$ to have $n$ repeated eigenvalues [32]
}

effects, which are constituted in form of loop-like notches in the circular or elliptic shapes of curves. For decreasing values of the second mode damping, abrupt energy exchanges between the modes induce non-periodic behavior.

3) Remarks on steady-state oscillations: Compared to oscillations induced by a human operator, the bang-bang regulator achieves ideal cyclic motions. While phase plots depicted in Fig. 8 deviate from ideal closed paths within a certain "error band", stable steady state motions in Fig. 9 display single, exactly closed curves for each joint coordinate. It remains open to further research, if the humans behavior is due to control imprecisions or has some other benefits.

\section{E. Summary}

Under specific conditions considered in this work, simulation results demonstrate that the modal parameters, i.e. eigenfrequency and damping, for the linearization of the system already provide a hint about the periodic behavior of this type of strongly nonlinear systems. Best preconditions for cyclic movements are different eigenfrequencies and different modal damping. This case applies to the robotic VIA arm in the absence of coupling springs. Therefore a simple controller is able to stabilize cyclic movement. Furthermore, the intrinsic system behavior is tending to first mode motions, even for similar eigenfrequencies, as long as the first eigenmode is weakly damped and the second eigenmode is strongly damped ${ }^{7}$.

\section{EXPERIMENTS: CONTROLLED OSCILLATIONS FOR A REAL VIA ROBOT ARM}

In the following, the insights obtained from simulations and the developed controller are experimentally tested and verified. Therefore, we used the first four variable impedance actuator joints of the DLR Hand Arm System. As the described analysis considers two joints, the robotic arm was configured such that only two joint axes (joint 2 and 4) were parallel and therefore coupled. Two initial configurations have been tested: \#1 $\boldsymbol{q}_{\mathrm{e}}=(0,0) \mathrm{rad}$ and $\# 2 \boldsymbol{q}_{\mathrm{e}}=(-0.27,0.64) \mathrm{rad}$. \#1 corresponds to an outstretched configuration, where the arm pointed in the direction of the gravity vector (down to the floor). Thereby, condition (12), given in Section III, is trivially fulfilled, i.e. $\boldsymbol{\theta}_{\mathrm{e}} \approx(0,0) \mathrm{rad}$. In the case \#2, the condition (12) requires $\boldsymbol{\theta}_{\mathrm{e}}=(-0.35,0.7) \mathrm{rad}$. Thus, for the experiments the robotic arm structurally corresponded to the system analyzed in the last sections, except that gravitational effects are included.

The bang-bang regulator (11) was used to generate the desired motor position of the first joint, while the measured spring torque of the same joint was the input of the controller. The desired motor position of the second joint (and all other joints not involved in motions) were constant. Since motor positions are not directly accessible, a motor PD controller tracked the desired trajectory (desired motor torques were commanded to the current controllers of the motors). Nonzero

\footnotetext{
${ }^{7}$ A generalization of the notion of eigenfrequencies and damping for the nonlinear case would be desirable here. In absence of such definitions in the literature, we observed that the values for the linearization of the system provide already a substantial amount of information.
} 


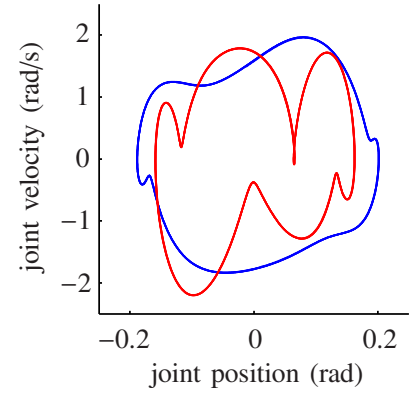

(a) \#1 $\boldsymbol{q}_{\mathrm{e}} \approx \boldsymbol{\theta}_{\mathrm{e}}=(0,0) \mathrm{rad}$
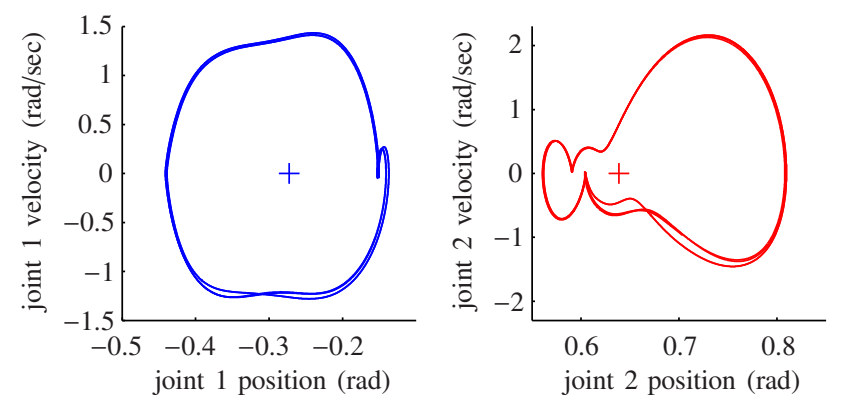

(b) \#2 $\boldsymbol{q}_{\mathrm{e}}=(-0.27,0.64) \mathrm{rad} ; \boldsymbol{\theta}_{\mathrm{e}}=(-0.35,0.7) \mathrm{rad}$

Fig. 11. Phase plots of joint motion $\boldsymbol{q}(t)$ vs. $\dot{\boldsymbol{q}}(t)$ obtained from experiments on the DLR Hand Arm System. The bang-bang controller (11) is connected to the first joint. Approximately, four cycles in the steady-state phase of oscillations are depicted. Joint positions are sampled at $1 \mathrm{kHz}$ and low-pass filtered (cut-off frequency $10 \mathrm{~Hz}$ ) before deriving the joint velocities, numerically.

initial conditions were set manually by pushing the robot by hand.

Phase plots of joint motions $\boldsymbol{q}(t)$ vs. $\dot{\boldsymbol{q}}(t)$ are depicted in Fig. 11. Here, approximately four periods in the stationary phase of oscillations are plotted. Furthermore, joint velocities are derived from measured and low-pass filtered joint positions (10 Hz cut-off frequency).

\#1: The shape of closed paths obtained by experiments is similar to simulations (cf. Fig. 9). The modal properties of the linearized system (in the initial configuration) are about $\omega=(6.6,34.1) \mathrm{rad} / \mathrm{s}$ for eigenfrequencies and $\xi=(0.03,0.28)$ for modal damping factors resulting from the natural, low damping in the spring mechanism.

\#2: As can be seen from Fig. 11(b) the equilibrium point is not in the center of the closed paths. This is as in the initial configuration the (nonlinear) springs are already elongated due to the gravity forces. Even in that case the resulting motion is periodic. Moreover, the modal properties of the linearized system (in the initial configuration) are slightly different compared to $\# 1: \omega=(8.0,35.0) \mathrm{rad} / \mathrm{s}$ and $\xi=(0.03,0.2)$.

As a consequence of eigenfrequency and modal damping distributions also the real robotic system tends to coordinated cyclic motions, while a simple controller is able to stabilize these oscillations. Thus one can observe that a planar, two joint VIA arm with approximately human like dimensions naturally fulfills the conditions for stable cyclic motions.

\section{Simulation Study based on EXPerimental data of the} HUMAN ARM

VIA robotic arms are strongly inspired by the biological musculo-skeletal system. Therefore, we can expect that also the human arm is predestined to execute such cyclic motions. In this section we verify this interesting hypothesis. Instead of accessing human motor control signals, mechanical properties of the human arm are identified and then considered in simulation, as for the VIA robotic arm in Section V.

\section{A. Estimation of mechanical properties of the human arm}

The method utilized here is described in [14] in detail. Thus, only a brief introduction of the method and results will be presented in the following.

1) Model of the musculo-skeletal system: The musculoskeletal system of the human arm is modeled by a slight generalization of the dynamic equations (3):

$$
\boldsymbol{M}(\boldsymbol{q}) \ddot{\boldsymbol{q}}+\boldsymbol{C}(\boldsymbol{q}, \dot{\boldsymbol{q}}) \dot{\boldsymbol{q}}+\frac{\partial U_{g}(\boldsymbol{q})}{\partial \boldsymbol{q}}=\boldsymbol{\tau}+\boldsymbol{\tau}_{\mathrm{ext}},
$$

where forces generated by muscles are introduced by joint torques

$$
\tau=-\boldsymbol{h}(\boldsymbol{q}, \dot{\boldsymbol{q}}, \boldsymbol{a})
$$

Similar to $\boldsymbol{\theta}$ in the case of VIA robots, the vector of muscle activations $\boldsymbol{a}$ can be seen as control input to the musculoskeletal system. A detailed description of (20) is beyond the scope of this paper $^{8}$; consequently we assume that the map (20) is continuous and we linearize it w.r.t. the equilibrium point $\boldsymbol{x}_{0}:=(\boldsymbol{q}(t=0), \dot{\boldsymbol{q}}(t=0), \boldsymbol{a}(t=0))$ :

$$
\begin{aligned}
\boldsymbol{h}^{\star}= & \underbrace{\left.\boldsymbol{h}\right|_{x_{0}}}_{\tau_{0}}+\underbrace{\left.\frac{\partial \boldsymbol{h}(\boldsymbol{q}, \dot{\boldsymbol{q}}, \boldsymbol{a})}{\partial \boldsymbol{q}}\right|_{\boldsymbol{x}_{0}}}_{\boldsymbol{K}_{0}} \tilde{\boldsymbol{q}}+\underbrace{\left.\frac{\partial \boldsymbol{h}(\boldsymbol{q}, \dot{\boldsymbol{q}}, \boldsymbol{a})}{\partial \dot{\boldsymbol{q}}}\right|_{x_{0}}}_{\boldsymbol{D}_{0}} \dot{\tilde{\boldsymbol{q}}} \\
& +\left.\frac{\partial \boldsymbol{h}(\boldsymbol{q}, \dot{\boldsymbol{q}}, \boldsymbol{a})}{\partial \boldsymbol{a}}\right|_{\boldsymbol{x}_{0}} \tilde{\boldsymbol{a}}+\ldots
\end{aligned}
$$

Herein $\tau_{0}$ is a vector of static joint torques at the equilibrium state $\boldsymbol{x}_{0}$. The Jacobians $\boldsymbol{K}_{0}$ and $\boldsymbol{D}_{0}$ are the positive definite and symmetric stiffness and damping matrices, respectively. Furthermore, $\tilde{\boldsymbol{q}}=\boldsymbol{q}-\boldsymbol{q}_{0}$ and $\tilde{\boldsymbol{a}}=\boldsymbol{a}-\boldsymbol{a}_{0}$ are small deviations. For the last term on the right hand side of (21) it is assumed that $\tilde{\boldsymbol{a}} \approx \mathbf{0}$, i.e. muscle activations remain constant. This is fulfilled by choosing proper experimental conditions [14]. Finally, the mechanical properties of the human arm (in the vicinity of $\boldsymbol{x}_{0}$ ) are determined by

$$
\boldsymbol{M}(\boldsymbol{q}, \zeta) \ddot{\boldsymbol{q}}+\boldsymbol{c}(\boldsymbol{q}, \dot{\boldsymbol{q}}, \zeta)+\boldsymbol{D}_{0} \dot{\tilde{\boldsymbol{q}}}+\boldsymbol{K}_{0} \tilde{\boldsymbol{q}}=\tilde{\boldsymbol{\tau}}_{\mathrm{ext}}
$$

where $\zeta$ is a vector of constant, identifiable inertial parameters.

Notice that the model (22) is linear in the unknown parameters (cf. [35]); consequently $\boldsymbol{\zeta}, \boldsymbol{D}_{0}$ and $\boldsymbol{K}_{0}$ can be estimated by means of common, linear least squares methods.

\footnotetext{
${ }^{8}$ Muscle models are discussed e.g. in [33] and [34] in detail.
} 


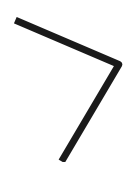

(a)

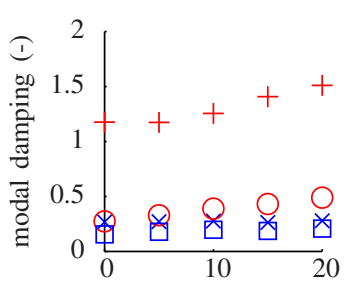

(e)

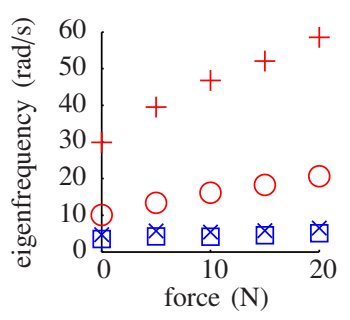

(i)

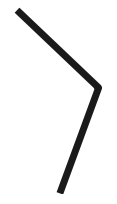

(b)

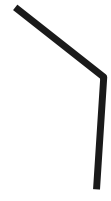

(c)

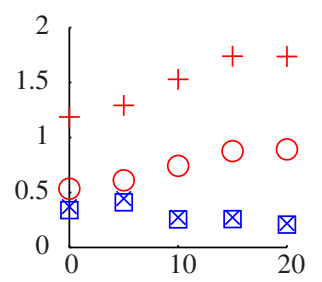

(g)

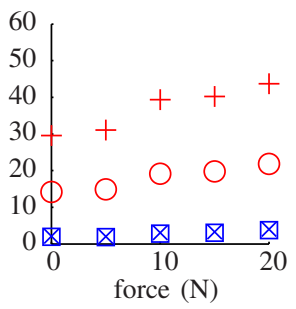

(k)

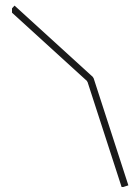

(d)

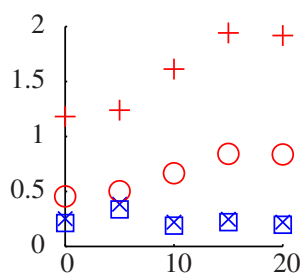

(f)

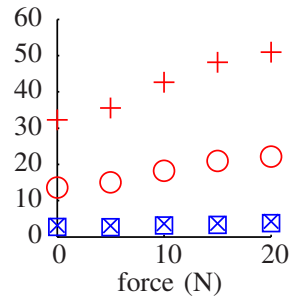

(j)

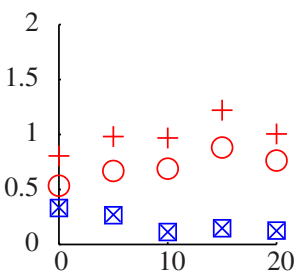

(h)

Fig. 12. Eigenfrequencies and normalized modal damping based on $\hat{\boldsymbol{\zeta}}, \hat{\boldsymbol{D}}_{0}$ and $\hat{\boldsymbol{K}}_{0}$. Columns of figures correspond to initial configurations depicted in the first row. The square marks represent $\xi_{1}\left(\boldsymbol{q}_{0}\right)$ or $\omega_{1}\left(\boldsymbol{q}_{0}\right)$ and the circle marks $\xi_{2}\left(\boldsymbol{q}_{0}\right)$ or $\omega_{2}\left(\boldsymbol{q}_{0}\right)$ (here $\boldsymbol{q}_{0}$ denotes the initial configuration). The X-marks represent $\xi_{1}(\mathbf{0})$ or $\omega_{1}(\mathbf{0})$ and the plus-marks $\xi_{2}(\mathbf{0})$ or $\omega_{2}(\mathbf{0})$.

2) Material and methods: The experimental setup used for parameter identification consist of the following parts: the main part is a position/torque-controlled light-weight robot, which is used to perturb the human arm and measure the arm position via the joint sensors (i.e., manipulator forward kinematics). The participant is fixed to a body-contoured seat to allow only motions of the subject's arm. Both, the robot and the seat are attached to a metal frame standing on the ground. To measure the interaction forces between the robot and the human arm, a six-axis JR3 force/torque sensor (FTS) is used. The robot end effector is connected to the human arm via a plastic cuff, which includes a metal beam supporting the arm against gravity. Both FTS and position data are recorded synchronously at the sampling rate of $1 \mathrm{kHz}$.

The whole experiment was performed in the human's transversal plane (i.e., perpendicular to the gravity vector). One healthy subject was tested. The subject's arm was held in one of four initial configurations by the robot. Furthermore, the subject was instructed to exert a predetermined force in distal direction with amplitudes $\left\|\boldsymbol{F}_{0}\right\| \in\{0,5,10,15,20\}(\mathrm{N})$, while the desired and actual force was displayed. After a random waiting time the robot had displaced the human arm in one of six randomly chosen (human arm's) joint space directions. For each initial configuration and force amplitude, data of 30 perturbations has been recorded (six directions, five times), to estimate one parameter set $\hat{\boldsymbol{\zeta}}, \hat{\boldsymbol{D}}_{0}$ and $\hat{\boldsymbol{K}}_{0}$.
3) Experimental results: To compare mechanical properties of human and robotic arms, estimated parameters are displayed in modal coordinates of the linearized system (see, Appendix A). Eigenvalues of estimated stiffness and damping matrices are linearly related to equilibrium torques $\boldsymbol{\tau}_{0}=\boldsymbol{J}\left(\boldsymbol{q}_{0}\right) \boldsymbol{F}_{0}$ (see, Appendix B). These relations indicate that joint stiffness and damping are independent of the initial configuration $\boldsymbol{q}_{0}$. This is also the case for the robotic arm (cf. (5)). Thus, estimated eigenfrequencies and modal damping are computed w.r.t. inertia matrices $\boldsymbol{M}\left(\boldsymbol{q}_{0}, \hat{\boldsymbol{\zeta}}\right)$ and $\boldsymbol{M}(\mathbf{0}, \hat{\boldsymbol{\zeta}})$, respectively (see, Fig. 12).

The difference between the first and second eigenfrequency monotonically increases for increasing forces exerted by the arm of the subject. Same tendencies can be observed for the damping of the first and second eigenmode. Even for the outstretched configuration $\boldsymbol{M}(\mathbf{0}, \hat{\boldsymbol{\zeta}})$ the first eigenmode is strongly under-critically and the second eigenmode is over-critically damped. These intrinsic damping properties are important requirements for coordinated, first mode cyclic motions.

\section{B. Simulated cyclic motion of the human arm}

To analyze the ability of the musculo-skeletal system for cyclic motions an assumption for the displacement dependent impedance term is required. The estimated stiffness matrix $\boldsymbol{K}_{0}$ determines the deflection / force relation in the vicinity of a stationary point, while the motion considered in this work requires large spring deflections. Therefore we assume a 


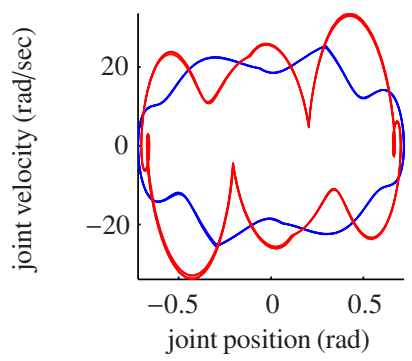

(a) $\xi=0.15,1.17 ; \omega=3.5,29.9$

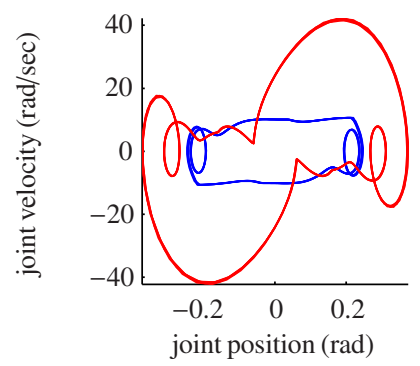

(b) $\xi=0.21,1.51 ; \omega=5.1,58.5$

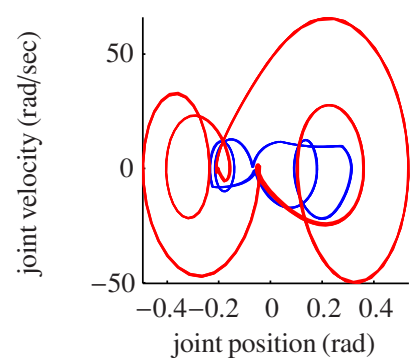

(c) $\xi=0.15,1.22 ; \omega=4.6,26.9$

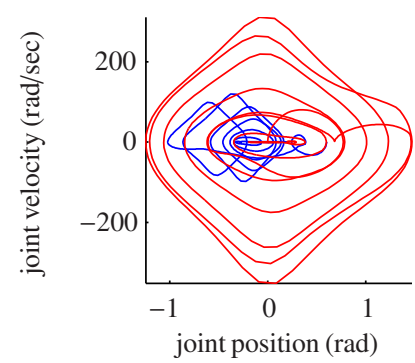

(d) $\xi=0.33,0.80 ; \omega=2.4,18.9$

Fig. 13. Phase plots of simulated motions, where the controller proposed in Section III is in the loop. Blue lines represent the pair $\left(q_{1}, \dot{q}_{1}\right)$ and red lines the pair $\left(q_{2}, \dot{q}_{2}\right)$. Simulations displayed corresponds to a human arm like mass distribution, where the spring and damping parameters are estimated from human measurements (cf. Fig. 12).

nonlinear, cubic characteristic (equal to the VIA robotic arm) to describe the (global) behavior of elasticities. The choice of such a progressive spring characteristic can be justified with the analysis done in [36]. Herein a static torque / angle relation for the elbow joint (measured during movements where the deflection velocity is negligible) was estimated, which can be approximated by a cubic polynomial function.

In simulation, estimated stiffness and damping matrices, and an average of estimated human arm like mass distributions are considered. These parameter sets correspond to modal properties shown in Fig. 12. In contrast to the procedure applied in Section V, (where eigenfrequencies and modal damping factors are assigned,) here, the experimentally estimated human stiffness and damping matrices are directly used, i.e. $\alpha_{1}=\hat{K}_{0,11}+\hat{K}_{0,12}, \alpha_{2}=\hat{K}_{0,22}+\hat{K}_{0,12}$, and $\alpha_{3}=-\hat{K}_{0,12}$ such that $\boldsymbol{K}_{0}\left(\alpha_{i}\right)=\hat{\boldsymbol{K}}_{0}$ (cf. (18)) and $\boldsymbol{D}=\boldsymbol{D}_{0}$. Note that eigenfrequencies $\omega_{2} / \omega_{1}>v_{\min }(\boldsymbol{M})$ can be also assigned with $\alpha_{3} \neq 0$ (stiffness coupling). Since the stiffness matrices, estimated for the human arm, are all coupled, but partially match the case of different eigenfrequencies, this study differs from what was done in Section V. Finally, it should be noted that all other simulation settings including the controller settings equal those considered in Section V.

As shown in Fig. 13 by simulation, also the musculoskeletal system has modal properties required for cyclic movement tendencies. Let us first discuss the limit cases: Phase plots of simulated motions depicted in Fig. 13(b) correspond to a large difference of eigenfrequencies and strongly over-critical damping of the second mode. In contrast, Fig. 13(d) corresponds to closer eigenfrequencies and closer modal damping. While in the former case notches are weakly developed, in the latter case a periodic motion is not achieved. Notice that stiffness and damping parameters corresponding to Fig. 13(b) were estimated in a kinematically non-singular configuration (i.e., due to large lever arms Cartesian forces act with high gains on joints) for the highest pretension force, while stiffness and damping parameters corresponding to Fig. 13(d) were estimated near a singular configuration (low joint torques for all Cartesian pretension forces). Since the stiffness (and damping) increase with increasing pretension forces (cf. Fig. 14 in the Appendix B), we can conclude that the musculo-skeletal system can be stiffen up by internal co-contraction of an- tagonistic muscles-resulting in best preconditions for cyclic motions. Furthermore, the cases depicted in Fig. 13(a) and (c) lie in between the limit cases, while phase plots displayed in Fig. 13(a) are most similar to simulated motions for the robotic arm in Fig. 9(a).

\section{Conclusion}

This work investigates main principles and requirements of cyclic motions in strongly nonlinear, compliantly actuated robotic systems. Starting with simple observations of humans controlling oscillations of serial type passive elastic systems, the human motor control and elastic system properties are analyzed. These experiments revealed that humans are able to control nonlinear, multi degree of freedom oscillations easily. Furthermore, extensive experiments with human in the loop simulations, hardware simulations, experiments on a real robotic system, and simulations of human arms are conducted. Afterwards, basic control principles observed in humans are transferred to compliantly actuated robotic arms and utilized to identify some basic system requirements for intrinsic cyclic motion tendencies.

The main contributions and findings are:

1) A simple and very robust control law to induce and stabilize multi degree of freedom oscillations that changes the intrinsic dynamics of the plant to a minimal extend and requires no model knowledge.

2) A qualitative analysis that identifies basic properties of compliantly actuated systems for cyclic motion tendencies. For desired motions in a specific mode, the damping of the system has to be such that oscillations excited in the remaining modes must decay faster than in the desired mode. In the case of serial type compliantly actuated systems, an eigenvalue analysis for the linearization of the system already provides a hint whether the system satisfies this damping property.

3) The human and the robotic arm DLR Hand Arm System are shown to fulfill these conditions.

The proposed bang-bang controller can excite and hold periodic motions for compliantly actuated robotic systems in a closed loop manner. Since the controller modifies the dynamics of the plant to a minimal extend, intrinsic oscillation properties of the system are exploited. In the current version 
of the approach, the stiffness preset of the VIA joints are kept constant. The controller parameters threshold and step amplitude can be tuned to adjust the amplitude and frequency of resulting oscillations in a certain range. However, for periodic tasks such as hammering or walking it could be desired to change the frequency and amplitude of oscillations in a wider range. To improve the adaptability of the resulting limit cycle to a certain task, the variability of the stiffness might be exploited. Thereby, the intrinsic resonance properties of the system might be varied by adjusting the characteristics of the springs.

In this work, the control law and the oscillation analysis are introduced for serial structured multi-body systems such as robotic and human arms, but the results can be straightforwardly transferred to robotic legs. For a legged system, the task is very clearly defined and the usage of these results are even of larger potential impact. Assuming the mechanical design is such that emerging oscillations correspond to useful gaits, then the proposed approach can be used to excite these cyclic movements. The stiffness variability and the initial joint configuration might be further used to tune the shape of the oscillation mode, e.g. to tune the frequency of the gait or to generate a transition from walking to running. Conversely, the analysis of the modal damping distribution might be used as rules of thumb for the mechanical design.

Furthermore, the proposed controller is introduced for a single-input single-output system (the controller is connected to a single joint). To improve the performance of the controlled system, the energy input to the system should be distributed to all of the (active) joints. The exploitation of the stiffness variability and the extension of the control method to the multiple-input multiple-output case are promising ongoing extensions of the current approach.

\section{ApPendix A}

\section{DEFINITIONS OF MODAL QUANTITIES}

In the following, we define modal quantities that are used as points of reference to discuss the oscillatory behavior of the system:

$$
\boldsymbol{M}(\boldsymbol{q}) \ddot{\boldsymbol{q}}+(\boldsymbol{C}(\boldsymbol{q}, \dot{\boldsymbol{q}})+\boldsymbol{D}) \dot{\boldsymbol{q}}+\frac{\partial U(\boldsymbol{\theta}, \boldsymbol{q})}{\partial \boldsymbol{q}}=\mathbf{0} .
$$

The definitions of these modal quantities are based on a instantaneous linearization of (A1) w.r.t. the system states at time instance $t_{0}$, i.e. $\boldsymbol{q}_{0}:=\boldsymbol{q}\left(t_{0}\right)$ and $\boldsymbol{\theta}_{0}:=\boldsymbol{\theta}\left(t_{0}\right)$. Furthermore, we assume in our definitions that $\dot{\boldsymbol{q}}_{0}=\dot{\boldsymbol{\theta}}_{0}=\mathbf{0}$.

Definition 1: The instantaneous linearization of (A1) at $\boldsymbol{q}_{0}$ and $\boldsymbol{\theta}_{0}$ is defined as

$$
\boldsymbol{M}_{0} \ddot{\boldsymbol{q}}+\boldsymbol{D}_{0} \dot{\boldsymbol{q}}+\boldsymbol{K}_{0} \boldsymbol{q}=\tau_{0},
$$

where $\boldsymbol{M}_{0}=\boldsymbol{M}\left(\boldsymbol{q}_{0}\right), \boldsymbol{D}_{0}=\boldsymbol{D}$,

$$
\boldsymbol{K}_{0}=\left.\frac{\partial^{2} U(\boldsymbol{\theta}, \boldsymbol{q})}{\partial \boldsymbol{q}^{2}}\right|_{\boldsymbol{\theta}=\boldsymbol{\theta}_{0}, \boldsymbol{q}=\boldsymbol{q}_{0}}, \text { and } \boldsymbol{\tau}_{0}=-\left.\frac{\partial U(\boldsymbol{\theta}, \boldsymbol{q})}{\partial \boldsymbol{q}}\right|_{\boldsymbol{\theta}=\boldsymbol{\theta}_{0}, \boldsymbol{q}=\boldsymbol{q}_{0}} .
$$

The definitions of the modal quantities are based on the following lemma:
Lemma 1 ( [37]): Given a symmetric and positive definite matrix $\boldsymbol{A} \in \mathbb{R}^{n \times n}$ and a symmetric matrix $\boldsymbol{B} \in \mathbb{R}^{n \times n}$. Then there exist a non-singular matrix $\boldsymbol{Q} \in \mathbb{R}^{n \times n}$ and a diagonal matrix $\boldsymbol{B}_{Q} \in \mathbb{R}^{n \times n}$, such that $\boldsymbol{Q}^{-T} \boldsymbol{Q}^{-1}=\boldsymbol{A}$ and $\boldsymbol{Q}^{-T} \boldsymbol{B}_{Q} \boldsymbol{Q}^{-1}=\boldsymbol{B}$.

Corollary 1: Given the matrices of Lemma 1 and a symmetric and positive definite matrix $C \in \mathbb{R}^{n \times n}$. Then there exist a diagonal matrix $\boldsymbol{C}_{Q} \in \mathbb{R}^{n \times n}$ such that $\boldsymbol{Q}^{-T} \boldsymbol{C}_{Q} \boldsymbol{Q}^{-1}=\boldsymbol{C}$ if $\boldsymbol{C}=c_{1} \boldsymbol{A}+c_{2} \boldsymbol{B}$.

Proof: The proof follows directly from Lemma 1, i.e.

$$
\boldsymbol{C}_{Q}=\boldsymbol{Q}^{T} \boldsymbol{C} \boldsymbol{Q}=\boldsymbol{Q}^{T}\left(c_{1} \boldsymbol{A}+c_{2} \boldsymbol{B}\right) \boldsymbol{Q}=c_{1} \boldsymbol{I}+c_{2} \boldsymbol{B}_{Q} .
$$

According to Lemma 1 , with $\boldsymbol{A} \triangleq \boldsymbol{M}_{0}$ and $\boldsymbol{B} \triangleq \boldsymbol{K}_{0}$,

$$
\ddot{z}+D_{\bmod }\left(\theta_{0}, q_{0}\right) \dot{z}+\Lambda\left(\theta_{0}, q_{0}\right) z=\tau_{z},
$$

represents the modal dynamics, corresponding to the linearized system (A2), where $\boldsymbol{z}=\boldsymbol{Q}\left(\boldsymbol{\theta}_{0}, \boldsymbol{q}_{0}\right)^{-1} \boldsymbol{q}$ are modal coordinates and $\boldsymbol{\Lambda}\left(\boldsymbol{\theta}_{0}, \boldsymbol{q}_{0}\right)$ is a diagonal matrix composed of real, positive eigenvalues (if $\boldsymbol{K}_{0}$ is positive definite). The modal damping matrix $\boldsymbol{D}_{\text {mod }}\left(\boldsymbol{\theta}_{0}, \boldsymbol{q}_{0}\right)=\boldsymbol{Q}\left(\boldsymbol{\theta}_{0}, \boldsymbol{q}_{0}\right)^{T} \boldsymbol{D}_{0} \boldsymbol{Q}\left(\boldsymbol{\theta}_{0}, \boldsymbol{q}_{0}\right)$ is assumed to be diagonal dominant. This implies that the condition of Corollary 1 is approximately satisfied: $\boldsymbol{D}_{0} \approx c_{1} \boldsymbol{M}_{0}+c_{2} \boldsymbol{K}_{0}$. In that case, the off-diagonal elements can be neglected ${ }^{9}$, i.e. $\boldsymbol{d}_{\text {mod }}\left(\boldsymbol{\theta}_{0}, \boldsymbol{q}_{0}\right)=\operatorname{diag}\left(\boldsymbol{D}_{\text {mod }}\left(\boldsymbol{\theta}_{0}, \boldsymbol{q}_{0}\right)\right)$. Furthermore, $\boldsymbol{\tau}_{z}=$ $\boldsymbol{Q}\left(\boldsymbol{\theta}_{0}, \boldsymbol{q}_{0}\right)^{T} \boldsymbol{\tau}_{0}$ represents a generalized, modal force.

Definition 2: The eigenfrequency of the $i$-th mode is defined as

$$
\omega_{i}\left(\boldsymbol{\theta}_{0}, \boldsymbol{q}_{0}\right)=\sqrt{\operatorname{diag}\left(\boldsymbol{\Lambda}\left(\boldsymbol{\theta}_{0}, \boldsymbol{q}_{0}\right)\right)_{i}} .
$$

Definition 3: The normalized damping factor of the $i$-th mode is defined as

$$
\xi_{i}\left(\boldsymbol{\theta}_{0}, \boldsymbol{q}_{0}\right)=\frac{d_{\mathrm{mod}, i}\left(\boldsymbol{\theta}_{0}, \boldsymbol{q}_{0}\right)}{2 \omega_{i}\left(\boldsymbol{\theta}_{0}, \boldsymbol{q}_{0}\right)}
$$

Using the Definitions 2 and 3, the modal dynamics (A3), corresponding to the linearized system (A2), yields:

$$
\ddot{z}_{i}+2 \xi_{i}\left(\boldsymbol{\theta}_{0}, \boldsymbol{q}_{0}\right) \omega_{i}\left(\boldsymbol{\theta}_{0}, \boldsymbol{q}_{0}\right) \dot{z}_{i}+\omega_{i}\left(\boldsymbol{\theta}_{0}, \boldsymbol{q}_{0}\right)^{2} z_{i}=\tau_{z_{i}} .
$$

Definition 4: The damping matrix $\boldsymbol{D}$ that specifies the viscous damping force $\boldsymbol{D} \dot{\boldsymbol{q}}$ in (A1) is referred to as physical damping. Given the desired modal damping factors $\xi_{i}$ and eigenfrequencies $\omega_{i}\left(\boldsymbol{\theta}_{0}, \boldsymbol{q}_{0}\right)$ (resulting from $\boldsymbol{K}_{0}$ and $\boldsymbol{M}_{0}$ of the linearized system (A2)), the corresponding, constant, physical damping matrix is defined as

$$
\boldsymbol{D}=2 \boldsymbol{Q}\left(\boldsymbol{\theta}_{0}, \boldsymbol{q}_{0}\right)^{-T} \operatorname{diag}\left(\xi_{i} \omega_{i}\left(\boldsymbol{\theta}_{0}, \boldsymbol{q}_{0}\right)\right) \boldsymbol{Q}\left(\boldsymbol{\theta}_{0}, \boldsymbol{q}_{0}\right)^{-1} .
$$

In the following, we tread the assignment of desired eigenfrequencies corresponding to the linearized, conservative system (cf. (A2))

$$
\boldsymbol{M}_{0} \ddot{\boldsymbol{q}}+\boldsymbol{K}_{0} \boldsymbol{q}=\mathbf{0}
$$

${ }^{9}$ Note, for the linear system (A2) an exact modal decomposition is proposed in [30] and will not be further detailed for simplicity. 
Therefore, we consider the following problem $^{10}$ :

Problem 1: Given a symmetric and positive definite matrix $\boldsymbol{M} \in \mathbb{R}^{n \times n}$ and $n$ desired eigenfrequencies $\omega_{i}$, find a real, symmetric, and positive definite matrix $\boldsymbol{K}_{0}$, such that

$$
\operatorname{det}\left(-\lambda \boldsymbol{M}+\boldsymbol{K}_{0}\right)=\prod_{i=1}^{n}\left(\lambda-\omega_{i}^{2}\right)
$$

holds.

Definition 5: If a matrix $\boldsymbol{K}_{0}$ can be found that solve the Problem 1, the corresponding eigenfrequencies are referred to as assigned eigenfrequencies of the linearized system (A2) (at the point $\left.\left(\boldsymbol{\theta}_{0}, \boldsymbol{q}_{0}\right)\right)$.

Solving Problem 1 involves dependencies in the choice of $\omega_{i}$ and the structure of $\boldsymbol{K}_{0}$. Multiple eigenfrequencies $\omega_{i}$ (multiplicity $n$ ) require the stiffness matrix $\boldsymbol{K}_{0}$ to be fully coupled [32], while the stiffness matrix of most VIA robot arms is diagonal.

In the following, the case $n=2$ is worked out. Therefore, a nonlinear coupled spring function is derived from the potential function:

$$
\begin{aligned}
U(\theta, \boldsymbol{q})= & \frac{1}{2} \alpha_{1}\left(q_{1}-\theta\right)^{2}+\frac{1}{4} \beta_{1}\left(q_{1}-\theta\right)^{4}+\frac{1}{2} \alpha_{2} q_{2}^{2}+\frac{1}{4} \beta_{2} q_{2}^{4} \\
& +\frac{1}{2} \alpha_{3}\left(q_{2}-\left(q_{1}-\theta\right)\right)^{2}+\frac{1}{4} \beta_{3}\left(q_{2}-\left(q_{1}-\theta\right)\right)^{4}
\end{aligned}
$$

where the negative gradient:

$$
\begin{aligned}
-\left(\frac{\partial U(\theta, \boldsymbol{q})}{\partial \boldsymbol{q}}\right)^{T}= & \boldsymbol{\psi}(\theta, \boldsymbol{q}), \\
\psi_{1}(\theta, \boldsymbol{q})= & -\alpha_{1}\left(q_{1}-\theta\right)-\beta_{1}\left(q_{1}-\theta\right)^{3} \\
& +\alpha_{3}\left(q_{2}-\left(q_{1}-\theta\right)\right)+\beta_{3}\left(q_{2}-\left(q_{1}-\theta\right)\right)^{3}, \\
\psi_{2}(\theta, \boldsymbol{q})= & -\alpha_{2} q_{2}-\beta_{2} q_{2}^{3} \\
& -\alpha_{3}\left(q_{2}-\left(q_{1}-\theta\right)\right)-\beta_{3}\left(q_{2}-\left(q_{1}-\theta\right)\right)^{3}
\end{aligned}
$$

represents the force field and the negative Jacobian:

$$
\begin{aligned}
-\frac{\partial \boldsymbol{\psi}(\theta, \boldsymbol{q})}{\partial \boldsymbol{q}} & =\boldsymbol{K}(\theta, \boldsymbol{q}), \\
K_{11}(\theta, \boldsymbol{q}) & =\alpha_{1}+3 \beta_{1}\left(q_{1}-\theta\right)^{2}+\alpha_{3}+3 \beta_{3}\left(q_{2}-\left(q_{1}-\theta\right)\right)^{2}, \\
K_{22}(\theta, \boldsymbol{q}) & =\alpha_{2}+3 \beta_{2} q_{2}^{2}+\alpha_{3}+3 \beta_{3}\left(q_{2}-\left(q_{1}-\theta\right)\right)^{2}, \\
K_{12}(\theta, \boldsymbol{q}) & =K_{21}(\theta, \boldsymbol{q})=-\alpha_{3}-\beta_{3}\left(q_{2}-\left(q_{1}-\theta\right)\right)^{2},
\end{aligned}
$$

the stiffness matrix.

Problem 1 is solved for $\alpha_{i}$ by substituting the linearized stiffness matrix

$$
\boldsymbol{K}_{0}=\left[\begin{array}{cc}
\alpha_{1}+\alpha_{3} & -\alpha_{3} \\
-\alpha_{3} & \alpha_{2}+\alpha_{3}
\end{array}\right],
$$

in (A9) and equating powers of $\lambda$ :

$$
\begin{aligned}
-\frac{\operatorname{det}\left(-\boldsymbol{M}+\boldsymbol{K}_{0}\right)-\operatorname{det}(\boldsymbol{M})-\operatorname{det}\left(\boldsymbol{K}_{0}\right)}{\operatorname{det}(\boldsymbol{M})} & =\omega_{1}^{2}+\omega_{2}^{2}, \\
\frac{\operatorname{det}\left(\boldsymbol{K}_{0}\right)}{\operatorname{det}(\boldsymbol{M})} & =\omega_{1} \omega_{2} .
\end{aligned}
$$

${ }^{10}$ This problem is similar to pole placement for linear state-feedback control (cf. [38]).
We obtain quadratic equations in powers of $\alpha_{i}$, which are solvable for $\alpha_{1}$ and $\alpha_{2}$, while $\alpha_{3}$ is a free parameter:

$$
\begin{aligned}
\alpha_{1}= & \frac{1}{2 M_{22}}\left(\left(\omega_{1}^{2}+\omega_{2}^{2}\right) \operatorname{det}(\boldsymbol{M})-2\left(M_{22}+M_{12}\right) \alpha_{3} \pm \sqrt{\mu}\right), \\
\alpha_{2}= & \frac{1}{2 M_{11}}\left(\left(\omega_{1}^{2}+\omega_{2}^{2}\right) \operatorname{det}(\boldsymbol{M})-2\left(M_{11}+M_{12}\right) \alpha_{3} \pm \sqrt{\mu}\right), \\
\mu= & \left(\omega_{1}^{2}-\omega_{2}^{2}\right)^{2} \operatorname{det}(\boldsymbol{M})^{2}-4 \omega_{1}^{2} \omega_{2}^{2} M_{12}^{2} \operatorname{det}(\boldsymbol{M}) \\
& -4 \operatorname{det}(\boldsymbol{M})\left(M_{12}\left(\omega_{1}^{2}+\omega_{2}^{2}\right)+\alpha_{3}\right) \alpha_{3} .
\end{aligned}
$$

The spring constants $\alpha_{1}$ and $\alpha_{2}$ have to be real. Additionally, the stiffness matrix $\boldsymbol{K}_{0}$ has to be positive definite; consequently $\alpha_{3}$ has to be chosen such that the discriminant $\mu \geq 0$ and the minor eigenvalue of the stiffness matrix $\min \left(\operatorname{eig}\left(\boldsymbol{K}_{0}\right)\right)>0$.

As a consequence of the coupled mass matrix, the stiffness matrix has to be non-diagonal (i.e., $\alpha_{3} \neq 0$ ) if the ratio of the assigned eigenfrequencies $v=\omega_{2} / \omega_{1}$ undercuts the greatest lower bound $v_{\min }(\boldsymbol{M})$. This property can be proven by substituting $\alpha_{3}=0$ in (A18) and solving for the ratio $\omega_{2} / \omega_{1}{ }^{11}$ :

$$
v_{\min }(\boldsymbol{M})=\frac{\sqrt{\operatorname{det}(\boldsymbol{M})+2 M_{12}^{2}+2 M_{12} \sqrt{M_{12}^{2}+\operatorname{det}(\boldsymbol{M})}}}{\sqrt{\operatorname{det}(\boldsymbol{M})}} .
$$

\section{APPENDix B}

STIFFNESS AND DAMPING OF THE MUSCULO-SKELETAL SYSTEM

The eigenvalues of joint stiffness and damping matrices are linearly related to the equilibrium torques $\tau_{0}$. This is validated by means of linear regression based on the model

$$
a_{i, 0}+a_{i, 1} \tau_{0,1}+a_{i, 2} \tau_{0,2}=\lambda_{i},
$$

where $a_{i, j}$ are constant coefficients. $\boldsymbol{\tau}_{0}=\boldsymbol{J}(\boldsymbol{q})^{T} \boldsymbol{F}_{0}$ is a vector of equilibrium joint torques resulting from initial forces $\boldsymbol{F}_{0}$. Estimated eigenvalues and approximations are displayed in Fig. 14. The quasi-linear relation indicates that joint visco elasticities of the musculo-skeletal system are independent of the limb configuration.

\section{ACKNOWLEDGMENT}

This work has been partially funded by the European Commission's Sixth Framework Programme as part of the project SAPHARI (grant number 287513). Additionally, the authors would like to thank Jordi Artigas for providing the force feedback device.

\section{REFERENCES}

[1] M. Grebenstein and P. v. d. Smagt, "Antagonism for a highly anthropomorphic hand-arm system," Advanced Robotics, vol. 22, no. 1, pp. 39-55, 2008.

[2] A. Albu-Schäffer, O. Eiberger, M. Fuchs, M. Grebenstein, S. Haddadin, C. Ott, A. Stemmer, T. Wimbck, S. Wolf, C. Borst, and G. Hirzinger, "Anthropomorphic soft robotics from torque control to variable intrinsic compliance," in Robotics Research, ser. Springer Tracts in Advanced Robotics, C. Pradalier, R. Siegwart, and G. Hirzinger, Eds. Springer, 2011, vol. 70, pp. 185-207.

${ }^{11}$ For the polynomial of degree four there exist four solutions, where only one solution is real and positive. 


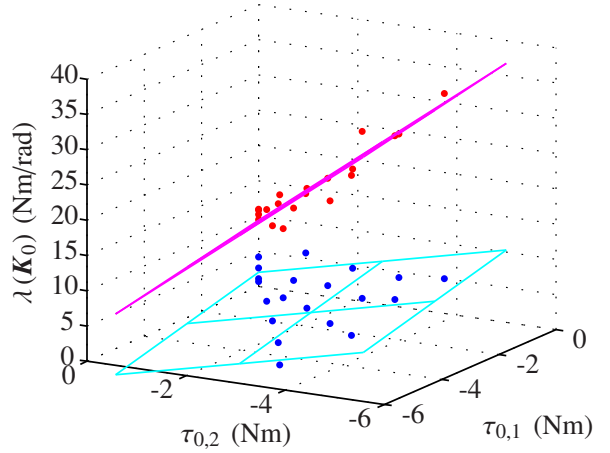

(a) Stiffness: first eigenvalues $R^{2}=0.637$, second eigenvalues $R^{2}=0.946$.

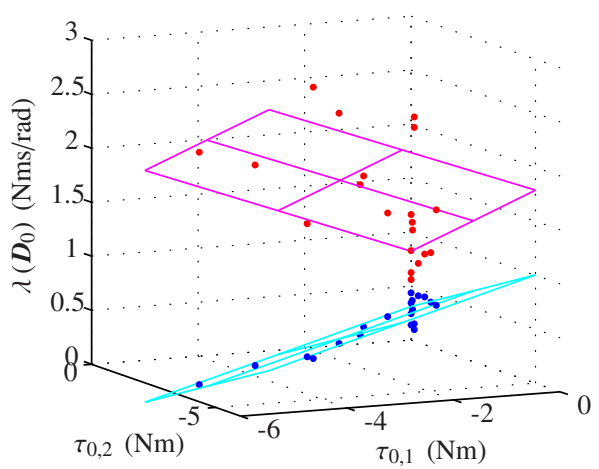

(b) Damping: first eigenvalues $R^{2}=0.804$, second eigenvalues $R^{2}=0.654$.

Fig. 14. Eigenvalues of joint stiffness and damping matrices estimated at human arm in four different configurations for five levels of initial forces (resulting equilibrium torques $\tau_{0}$ depend on configuration). Surfaces represent linear approximations by means of the model (B1). The coefficients of determination $R^{2}$ indicate the goodness of the fits.

[3] K. C. Galloway, J. E. Clark, M. Yim, and D. E. Koditschek, "Experimental investigations into the role of passive variable compliant legs for dynamic robotic locomotion," in Proc. IEEE Int. Conf. on Robotics and Automation, 2011.

[4] L. C. Visser, S. Stramigioli, and A. Bicchi, "Embodying desired behavior in variable stiffness actuators," in IFAC Congress of the International Federation of Automatic Control, 2011.

[5] M. Garabini, A. Passaglia, F. A. W. Belo, P. Salaris, and A. Bicchi, "Optimality principles in variable stiffness control: The vsa hammer." in IEEE/RSJ Int. Conf. on Intelligent Robots and Systems, 2011, pp. 3770-3775.

[6] D. J. Braun, M. Howard, and S. Vijayakumar, "Exploiting variable stiffness in explosive movement tasks," in Robotics: Science and Systems, 2011.

[7] S. Haddadin, F. Huber, and A. Albu-Schäffer, "Optimal control for exploiting the natural dynamics of variable stiffness robots," in IEEE Int. Conf. on Robotics and Automation, 2012.

[8] A. J. Ijspeert, "A connectionist central pattern generator for the aquatic and terrestrial gaits of a simulated salamander," Biological Cybernetics, vol. 84, pp. 331-348, 2001.

[9] J. Buchli and A. J. Ijspeert, "Distributed central pattern generator model for robotics application based on phase sensitivity analysis," in BioADIT'04, 2004, pp. 333-349.

[10] A. J. Ijspeert and A. Crespi, "Online trajectory generation in an amphibious snake robot using a lamprey-like central pattern generator model," in IEEE Int. Conf. on Robotics and Automation, 2007, pp. 262 268.

[11] D. Lakatos, F. Petit, and A. Albu-Schäffer, "Nonlinear oscillations for cyclic movements in variable impedance actuated robotic arms," in IEEE Int. Conf. on Robotics and Automation, 2013.

[12] A. H. Nayfeh and D. T. Mook, Nonlinear Oscillations. John Wiley \& Sons, 1979
[13] J. Guckenheimer and P. Holmes, Nonlinear Oscillations, Dynamical Systems, and Bifurcations of Vector Fields. Springer, 1983.

[14] D. Lakatos, F. Petit, and P. Smagt, "Conditioning vs. excitation time for estimating impedance parameters of the human arm," in IEEE-RAS Int. Conf. on Humanoid Robots (Humanoids), 2011, pp. 636-642.

[15] F. A. Mussa-Ivaldi, N. Hogan, and E. Bizzi, "Neural, mechanical, and geometric factors subserving arm posture in humans," The Journal of Neuroscience, vol. 5, pp. 2732-2743, 1985.

[16] T. Tsuji, P. G. Morasso, K. Goto, and K. Ito, "Human hand impedance characteristics during maintained posture," Biological Cybernetics, vol. 72, pp. 475-485, 1995.

[17] H. Gomi and M. Kawato, "Human arm stiffness and equilibrium-point trajectory during multi-joint movement," Biological Cybernetics, vol. 76, pp. 163-171, 1997.

[18] H. Gomi and R. Osu, "Task-dependent viscoelasticity of human multijoint arm and its spatial characteristics for interaction with environments," The Journal of Neuroscience, vol. 18, pp. 8965-8978, 1998.

[19] E. J. Perreault, R. F. Kirsch, and P. E. Crago, "Effects of voluntary force generation on the elastic components of endpoint stiffness," Experimental Brain Research, vol. 141, pp. 312-323, 2001.

[20] A. Albu-Schäffer, S. Wolf, O. Eiberger, S. Haddadin, F. Petit, and M. Chalon, "Dynamic modelling and control of variable stiffness actuators," in IEEE Int. Conf. on Robotics and Automation, 2010.

[21] A. Albu-Schäffer, C. Ott, and F. Petit, "Constructive energie shaping control for a class of euler-lagrange systems," in 10th Int. IFAC Symposium on Robot Control, 2012.

[22] C. Ott, Cartesian Impedance Control of Redundant and Flexible-Joint Robots, B. Siciliano and O. Khatib, Eds. Springer, 2008.

[23] P. Kokotovic, H. Khalil, and J. O'Reilly, Singular Perturbation Methods in Control: Analysis and Design. Academic Press, London, 1986.

[24] H. Khalil, Nonlinear Systems, 3rd edn. Prentice Hall, Englewood Cliffs, 2002.

[25] M. Grebenstein, A. Albu-Schäffer, T. Bahls, M. Chalon, O. Eiberger, W. Friedl, R. Gruber, S. Haddadin, U. Hagn, R. Haslinger, H. Höppner, S. Jörg, M. Nickl, A. Nothhelfer, F. Petit, J. Reill, N. Seitz, T. Wimböck, S. Wolf, T. Wüsthoff, and G. Hirzinger, "The DLR Hand Arm System," in Proc. IEEE Int. Conf. on Robotics and Automation, 2011.

[26] S. Wolf and G. Hirzinger, "A new variable stiffness design: Matching requirements of the next robot generation," in Proc. IEEE Int. Conf. on Robotics and Automation, 2008.

[27] A. H. Nayfeh, Perturbation Methods. John Wiley \& Sons, 1973.

[28] M. Pakdemirli, M. Karahan, and H. Boyaci, "Forced vibrations of strongly nonlinear systems with multiple scales Lindstedt Poincare method," Mathematical and Computational Applications, vol. 16, no. 4, pp. 879-889, 2011.

[29] D. Lakatos, G. Garofalo, F. Petit, C. Ott, and A. Albu-Schäffer, "Modal limit cycle control for variable stiffness actuated robots," in IEEE Int. Conf. on Robotics and Automation, 2013.

[30] S. Shaw and C. Pierre, "Normal modes for non-linear vibratory systems," Journal of Sound and Vibration, vol. 164, no. 1, pp. 85-124, 1993.

[31] J. Hoffer, R. Stein, M. Haugland, T. Sinkjaer, W. Durfee, A. Schwartz, G. Loeb, and C. Kantor, "Neural signals for command control and feedback in functional neuromuscular stimulation: a review." Journal of Rehabilitation Research and Development, vol. 33, no. 2, pp. 145-157, 1996.

[32] F. Petit, D. Lakatos, W. Friedl, and A. Albu-Schäffer, "Dynamic trajectory generation for serial elastic actuated robots," in 10th Int. IFAC Symposium on Robot Control, 2012.

[33] J. M. Winters and L. Stark, "Analysis of fundamental human movement patterns through the use of in-depth antagonistic muscle models," IEEE Transaction on Biomedical Engineering, vol. 32, pp. 826-839, 1985.

[34] _ "Muscle models: What is gained and what is lost by varying model complexity," Biological Cybernetics, vol. 55, pp. 403-420, 1987.

[35] W. Khalil and E. Dombre, Modeling, Identification and Control of Robots, 3rd ed. Bristol, PA, USA: Taylor \& Francis, Inc., 2002.

[36] R. Shadmehr and M. A. Arbib, "A mathematical analysis of the forcestiffness characteristics of muscles in control of a single joint system," Biological Cybernetics, vol. 66, pp. 463-477, 1992.

[37] R. Horn and C. Johnson, Matrix Analysis. Cambridge University Press, 1985.

[38] J. Kautsky, N. K. Nichols, and P. van Dooren, "Robust pole assignment in linear state feedback," International Journal of Control, vol. 41, no. 5, pp. 1129-1155, 1985. 


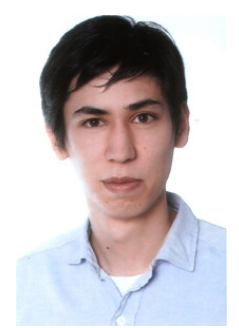

Dominic Lakatos received the Dipl.-Ing. degree in mechanical engineering from the University of Applied Sciences Munich, Germany, in 2011. Since 2011 he has been with the Institute of Robotics and Mechatronics, German Aerospace Center, Wessling, Germany. His main research interests include control of nonlinear systems, under-actuated mechanical systems, human and robotic dynamics, nonlinear oscillations, limit cycles, bio-inspired and legged robots.

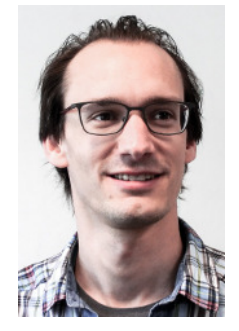

Florian Petit received the Dipl.-Ing. degree in electrical engineering from the Technical University of Munich (TUM), Munich, Germany, in 2008. He joined the Institute of Robotics and Mechatronics, German Aerospace Center (DLR), Wessling, Germany, in 2009. He is also member of the SensoryMotor Systems Laboratory, Swiss Federal Institute of Technology Zurich (ETHZ), Zurich, Switzerland, since 2012. His research interests include variable impedance robots and control, impedance and force control, and nonlinear systems and control.

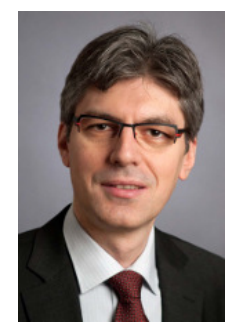

Alin Albu-Schäffer graduated in electrical engineering at the Technical University of Timisoara, in 1993 and got the PhD in automatic control from the Technical University of Munich in 2002. Since 2012 he is the head of the Institute of Robotics and Mechatronics at the German Aerospace Center, which he joint in 1995 as a $\mathrm{PhD}$ candidate. Moreover, he is a professor at the Technical University of Munich, holding the Chair for Sensorbased Robotic Systems and Intelligent Assistance Systems at the Computer Science Department. His personal research interests include robot design, modeling and control, nonlinear systems, flexible joint and variable compliance robots, impedance and force control, physical humanrobot interaction, bio-inspired robot design. 